DOI: 10.1002/((please add manuscript number))

Article type: Full Paper

\title{
A magnetocaloric composite based on molecular coolers and carbon nanotubes with enhanced thermal conductivity
}

Olivier Roubeau,* Eva Natividad, Marco Evangelisti, Giulia Lorusso and Elías Palacios

Dr. O. Roubeau, Dr. E. Natividad, Dr. M. Evangelisti, Dr. G. Lorusso, Dr. E Palacios Instituto de Ciencia de Materiales de Aragón (ICMA), CSIC and Universidad de Zaragoza, Plaza San Francisco s/n, 50009 Zaragoza, Spain

E-mail:roubeau@unizar.es

Dr. O. Roubeau, Dr. M. Evangelisti, Dr. G. Lorusso, Dr. E Palacios

Departamento de Física de la MateriaCondensada, Universidad de Zaragoza, C/ Pedro Cerbuna 12, 50009 Zaragoza, Spain

Dr. E. Natividad

Departamento de Ciencia y Tecnología de Materiales y Fluidos, Universidad de Zaragoza, María de Luna 3, 50018 Zaragoza, Spain

Keywords: magnetocalorics, molecular materials, cryogenicrefrigeration, thermal conductivity, carbon nanotubes

In spite of a remarkably large magnetocaloric effect at temperatures below ca. $10 \mathrm{~K}$, molecular coolers are yet to become excellent magnetic refrigerants. Their main limitation lies in the heat transport across molecules, expected to decrease drastically at cryogenic temperatures, although no experimental data are in fact available. Here, the thermal conductivity of the $\left[\mathrm{Gd}_{2}(\mathrm{OAc})_{6}\left(\mathrm{H}_{2} \mathrm{O}\right)_{4}\right] \cdot 4 \mathrm{H}_{2} \mathrm{O}$ molecular cooler is found to decrease from 0.02 $\mathrm{Wm}^{-1} \mathrm{~K}^{-1}$ at $153 \mathrm{~K}$ down to $3.2 \square \times \square 10^{4} \mathrm{Wm}^{-1} \mathrm{~K}^{-1}$ at $4.5 \mathrm{~K}$. For samples of different thicknesses, direct measurements of the magnetocaloric effect and numerical simulations corroborate how detrimental the poor thermal conductivity can be for magnetic refrigeration. As a way out, a hybrid composite material is formed by the efficient attachment of the carboxylate-bridged Gd(III) molecules onto oxidized multi-walled carbon nanotubes. The magnetocaloric properties of this composite show that its molecular component maintains its functionality, e.g., $\square \Delta S_{\mathrm{m}}=40.0 \mathrm{~J} \mathrm{~kg}^{-1} \mathrm{~K}^{-1}$ at $T=1.8 \mathrm{~K}$ and $\Delta T_{\mathrm{ad}}=12.8 \mathrm{~K}$ at $T=1.6 \mathrm{~K}$, both 
for $\Delta B=(7-0) T$. Notably, the thermal conductivity of oriented compositebuckypapers surpasses that of bulk $\left[\mathrm{Gd}_{2}(\mathrm{OAc})_{6}\left(\mathrm{H}_{2} \mathrm{O}\right)_{4}\right] \cdot 4 \mathrm{H}_{2} \mathrm{O}$ by a factor of 2-3 below $20 \mathrm{~K}$. Eventually, direct measurements of the magnetocaloric effecton the composite evidence the benefits arising from its higher thermal conductivity, in the form of lower temperatures reached. This improved performance validates the strategy aiming at hybrid composite materials based on molecular coolers and a material with good intrinsic thermal conductivity.

\section{Introduction}

Cryogenic cooling applications using the so-called adiabatic demagnetization process rely on the large magneto-caloric effect (MCE) of inorganic salts, originally used to attain sufficiently low temperatures as to allow the condensation of hydrogen and helium. ${ }^{[1]}$ Adiabatic demagnetization refrigerators (ADR) have proven to be alternatives to ${ }^{3} \mathrm{He}-{ }^{4} \mathrm{He}$ dilution fridges for ultra-low-temperature space applications. ${ }^{[2]}$ More generally, magnetic refrigeration at liquid-helium temperatures represents a valuable alternative to helium itself given the rareness of ${ }^{3} \mathrm{He}$ and the increased costs of both isotopes. ${ }^{[3]}$ This technology has been somewhat unchallenged due to the suitability of the materials used, ${ }^{[3,4]}$ and the niche type of most applications in low-temperature physics. There are however potential improvements in terms of the weight and processability of materials used. In particular, employing thin films for local on-chip cooling would be highly interesting in space applications and microrefrigeration, ${ }^{[5]}$ and it justifies the search for new materials. In this respect, the proposal that magnetic molecules with high spin state and high magnetic vs. non-magnetic element ratio can compete with commercially-used materials such as Gadolinium Gallium Garnet (GGG) is particularly appealing, and it has aroused much interest into the field of so-called molecular coolers. ${ }^{[6,7]}$ Although many 3d-Gd and purely Gd(III) medium-to-high nuclearity molecules have now been reported with large MCE, ${ }^{[7]}$ simple homometallicGd(III) compounds probably represent the ideal truly molecular coolers, as exemplified by gadolinium acetate tetrahydrate, 
$\left[\mathrm{Gd}_{2}(\mathrm{OAc})_{6}\left(\mathrm{H}_{2} \mathrm{O}\right)_{4}\right] \cdot 4 \mathrm{H}_{2} \mathrm{O}$ (hereafter denoted as $\left[\mathrm{Gd}_{2} \mathrm{ac}\right]$ ), a ferromagnetic molecular dinuclear compound with a volumetric entropy change $-\rho \Delta S_{\mathrm{m}}$ of $55 \mathrm{mJcm}^{-3} \mathrm{~K}^{-1}$ for an applied magnetic field change of $\Delta B=1 \mathrm{~T} \cdot{ }^{[8]}$ Promising molecular-based magnetocooling materials are also dense extended coordination frameworks, ${ }^{[9]}$ such as gadolinium formate, [Gd(HCOO) $\left.)_{3}\right]$, that with its $-\rho \Delta S_{\mathrm{m}}$ of $120 \mathrm{mJcm}^{-3} \mathrm{~K}^{-1}$ for $\Delta B=1 \mathrm{~T}$ even outperforms GGG. ${ }^{\text {[9a] }}$ Since long-range magnetic order limits efficient cooling below the ordering temperature, diluted paramagnetic salts such as cerium magnesium nitrate (CMN) or chromic potassium alun (CPA) are used commercially to attain ultra-low temperatures. ${ }^{[3]}$ These materials however present a significant magnetic anisotropy and a low refrigeration power, making molecular coolers interesting alternatives once again. For example, $\left[\mathrm{Gd}_{2} \mathrm{ac}\right]$ undergoes a phase transition to an antiferromagnetically ordered state at a temperature as low as $0.2 \mathrm{~K},{ }^{[8]}$ while Gd(III)-based polyoxometallates can remain paramagnetic down to ca. $10 \mathrm{mK} \cdot{ }^{[10]}$ Any of these molecular-based coolers is however likely to display an unfavorable thermal conductivity, as a result of the intermolecular and/or inorganic-organic interfaces in their structure, even in the case of extended frameworks. ${ }^{[11,12]}$ There are nevertheless no lowtemperature thermal conductivity data available so far for molecular-based coolers, while this is long-known to be a critical parameter for magnetic refrigerators. ${ }^{[13]}$ For on-chip microrefrigeration, the expected poor thermal conductivity may have only a limited negative effect since the cooling materials could be deposited as nano-layers on the substrate to be cooled, while maintaining their MCE.$^{[14]}$ For bulk applications, such as in ADRs, in which a low thermal conductivity would be definitively negative, a solution could be to design composite materials built on molecular coolers in combination with a good thermal conductor. The latter should obviously be light and most of all have a vanishing heat capacity in the temperature range of interest. Carbon-based nanomaterials such as carbon nanotubes (CNTs) thus appear as good candidates, ${ }^{[15]}$ provided a dense functionalization of their surface with molecular- 
based coolers is possible, either by covalent approaches or through strong non-covalent interactions. For such aim, truly molecular coolers seem more adequate, due to the flexibility of their binding to surfaces, as opposed to extended frameworks. An additional advantage is that the loss of crystalline order would then annihilate the probability of any magnetic order, thus opening cooling to lower temperature ranges. We report here the first experimental determination of the low-temperature thermal conductivity of a molecular-based cooler, [Gd $\mathrm{G}_{2} \mathrm{ac}$ ], which confirms the expected poor properties of this family of materials. As a first attempt to compensate this drawback, multi-walled CNTs are efficiently functionalized with [Gd $\left.\mathrm{G}_{2} \mathrm{ac}\right]$ molecules. The magneto-thermal performance of the resulting composite material is evaluated through bulk magnetization, heat capacity, thermal conductivity and direct measurements of the MCE.

\section{Results and Discussion}

\subsection{Composite elaboration, composition and structure}

A natural choice of molecular cooler for this study was $\left[\mathrm{Gd}_{2}(\mathrm{OAc})_{6}\left(\mathrm{H}_{2} \mathrm{O}\right)_{4}\right] \cdot 4 \mathrm{H}_{2} \mathrm{O}$, hereafter abbreviated $\left[\mathrm{Gd}_{2} \mathrm{ac}\right]$, for its high MCE, simplicity, ease of preparation and robustness. Then the strategy to efficiently cover a carbon nanomaterial with it logically contemplated the use of oxidized carbon nanotubes, and more specifically multi-walled carbon nanotubes (MWCNT). Indeed oxidation of carbon nanotubes, widely-used for their functionalization, results in numerous oxygen-rich functions on the outer surface of MWCNT, in particular carboxylic groups. ${ }^{[16,17]}$ These allow for both covalent attachment of the selected molecule, by replacing one of its terminal acetate ligand, as well as non-covalent strong interactions through multiple H-bonds. This strategy (Scheme 1) represents an efficient way to produce an intimate coating of CNT by molecular coolers, dense but thin, i.e. ideal for efficient cooling. The oxidized MWCNTs form stable solutions in water, in which $\left[\mathrm{Gd}_{2} \mathrm{ac}\right]$ is actually synthesized and highly soluble, a further advantage over more elegant covalent modifications 
of CNTs ${ }^{[17]}$ for the specific objective of this work, i.e. simple, efficient and cost-effective means, not requiring extreme conditions nor multi-step synthesis.

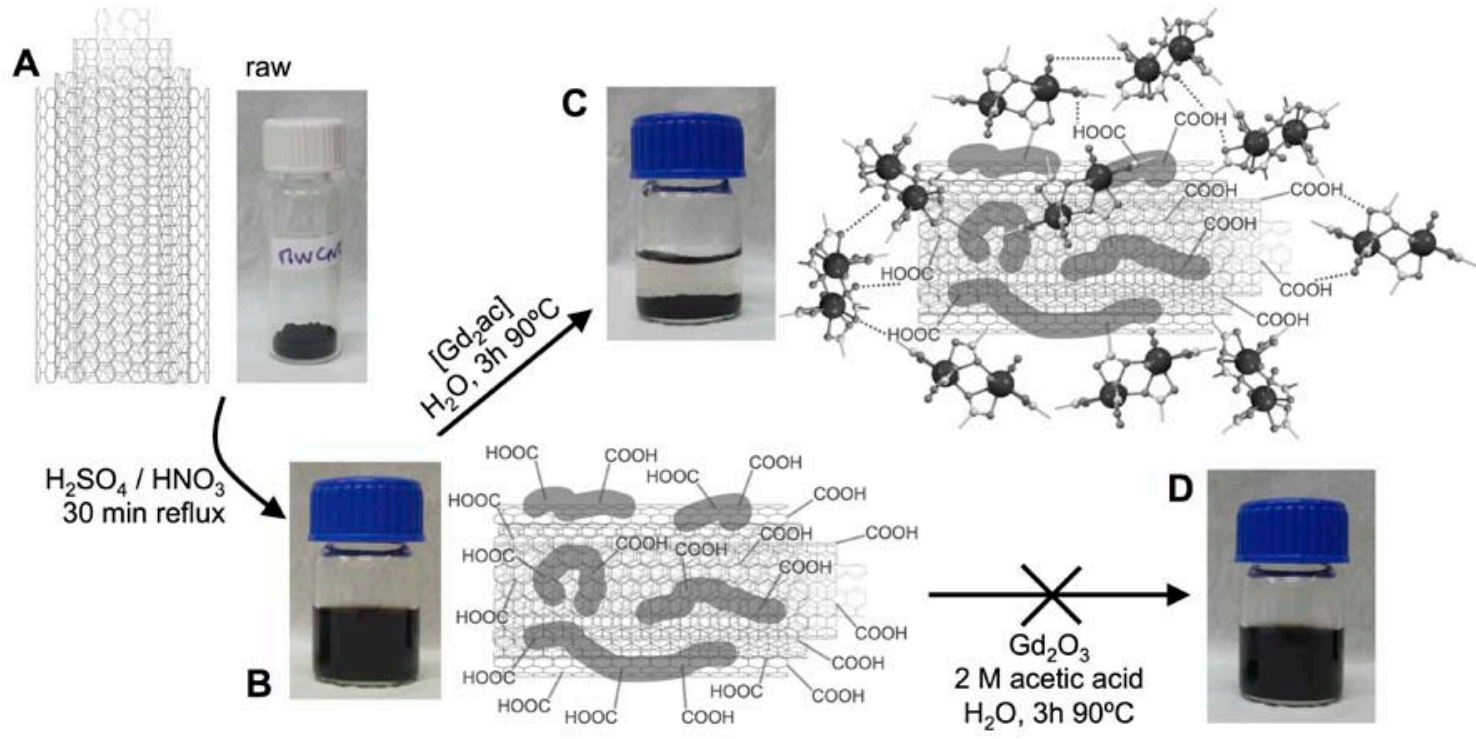

Scheme 1. Synthetic strategy. A: raw MWCNTs. B: oxidation of MWCNTs. C: reaction with preformed $\left[\mathrm{Gd}_{2} \mathrm{ac}\right]$ towards the elaboration of a MWCNT-[ $\left.\mathrm{Gd}_{2} \mathrm{ac}\right]$ composite material. The use of conditions to produce $\left[\mathrm{Gd}_{2} \mathrm{ac}\right]$ in-situ (D) proved ineffective (see text).

The used raw MWCNTs were dispersed in water using sodium dodecyl sulfate and tip sonication and characterized by TEM showing lack of agglomeration and diameters in the range 5-20 nm (see Figure S1), in agreement with the provided nominal values. These raw MWCNTs were then oxidized by warming the fine black powder in a 2:1 mixture of sulfuric and nitric acids, under vigorous stirring and for only $30 \mathrm{~min}$. After work-up, the resulting oxidized MWCNTs (MWCNT-ox) were recovered as a wet black cake and immediately redispersed in deionized water, forming a stable black dispersion (see Scheme 1, part B). Not drying the MWCNT-ox solid allows full and easy solubilisation, by avoiding the formation of large aggregates, an easier alternative to freeze-drying. ${ }^{[18]}$ Indeed, the formed dispersion is stable for long periods of time (months) and no significant aggregates are observed by optical microscopy of a drop of the dispersion in between glass slides. Here it should be emphasized that the work-up used purposely does not include any base treatment. The reason is that this treatment removes the polyaromatic and amorphous carbons that are known to result from the 
oxidation damage to the outer wall(s) of CNTs. ${ }^{[19,20]}$ These are known to be heavily covered with carboxylic and other oxygen-rich groups while being strongly bonded/stacked to the CNTs core. ${ }^{[19,20]}$ They are therefore very useful for our purpose, since the MWCNT-ox core is known to have fewer carboxylic functions on its walls, those being more dense on the MWCNT-ox open ends. Dry extracts of the stable stock dispersion give a concentration of $c a$. $2.7 \mathrm{~g} / \mathrm{L}$ of MWCNT-ox. Thermogravimetric analysis provides a measure of the oxygen-rich functions at ca. 6 wt. \% (Figure S2 and Table S1). Powder X-Ray Diffraction (PXRD) indicate the material maintains its multiwall structure (Figure S3), while TEM observations show that the MWCNT are not significantly cut through the oxidation process and that the average diameter of the MWCNT-ox remains similar to that of raw MWCNT (Figure 1 and S1 for raw MWCNTs). Also, the observed surface roughness confirms the presence of an outer layer of amorphous material. ${ }^{[19,20]}$
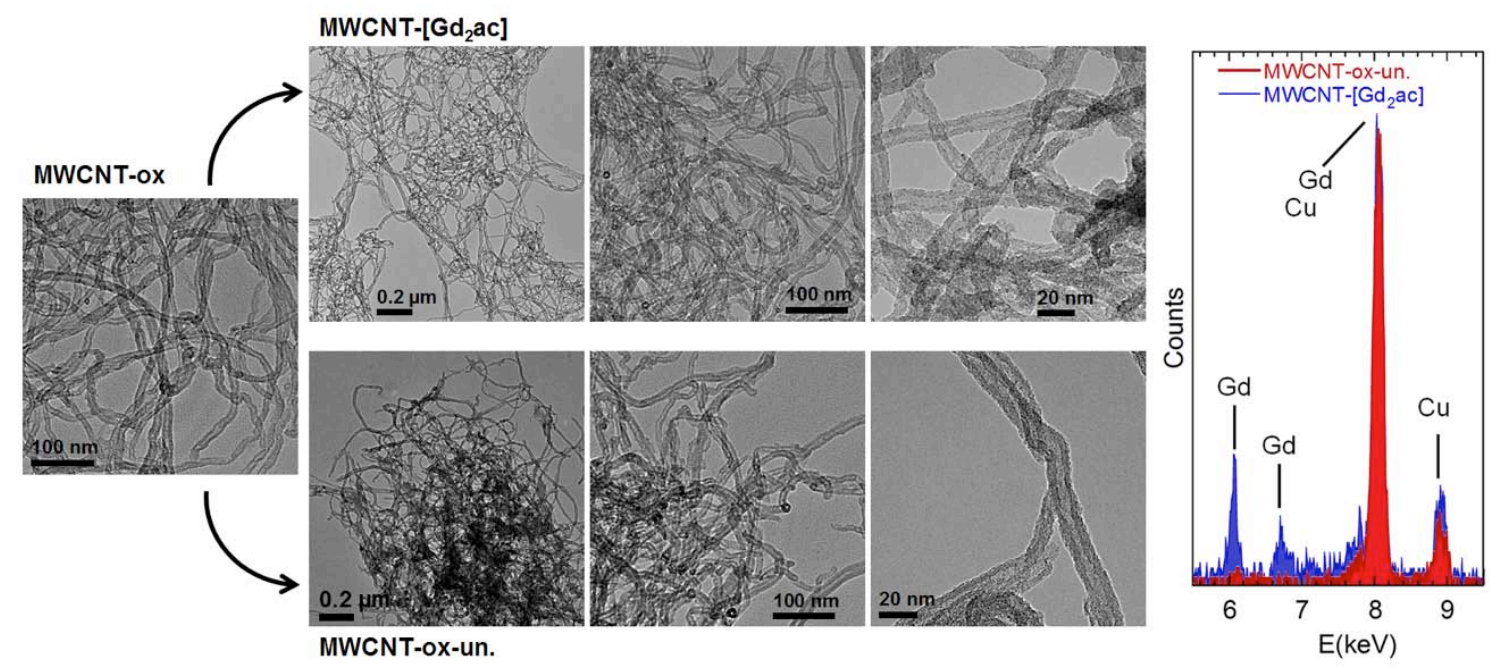

Figure 1. Representative TEM observations of MWCNT-ox (left), MWCNT-[Gd $\left.\mathrm{d}_{2} \mathrm{ac}\right]$ (middle-top) and the unmodified material (MWCNT-ox-un.) isolated upon reaction of MWCNT-ox with $\mathrm{Gd}_{2} \mathrm{O}_{3}$ and acetic acid (middle-bottom). Open ends observed in some images evidence the multi-wall structure. EDX analysis (right) showing respectively the presence and absence of Gd in the latter two materials. Copper from the TEM grid is also detected.

Functionalization of the MWCNT-ox was then attempted by two means (Scheme 1). First, a portion of the MWCNT-ox aqueous dispersion was reacted at $90{ }^{\circ} \mathrm{C}$ for 3 hours with 
preformed $\left[\mathrm{Gd}_{2} \mathrm{ac}\right]$ dissolved in little deionized water. The black dispersion almost immediately got poorer, clearly indicating the reaction of the oxygen-rich functions at the origin of the good dispersability of MWCNT-ox. The black solid recovered proved to be the targeted hybrid composite material, from here on MWCNT-[Gd $\mathrm{Gc}_{2} \mathrm{c}$, (see below and Scheme 1 , part C) and did not redisperse in water. Alternatively, the same white slurry of $\mathrm{Gd}_{2} \mathrm{O}_{3}$ in water/acetic acid as used for the synthesis of [ $\left.\mathrm{Gd}_{2} \mathrm{ac}\right]$ was mixed with a portion of the MWCNT-ox dispersion, under the exact same conditions, thus replicating the conditions used for the formation of $\left[\mathrm{Gd}_{2} \mathrm{ac}\right]$. In this case, the dispersion surprisingly remained stable, apparently indicating that no reaction involving MWCNT-ox took place. Indeed, the black solid recovered contains no Gd at all, easily re-disperses in water and thus appears to be unreacted MWCNT-ox (Scheme 1, part D). The necessity to use pre-formed $\left[\mathrm{Gd}_{2} \mathrm{ac}\right]$ as opposed to forming it in-situ is surprising and may be due to the fact the presence of the MWCNT-ox affects the formation of $\left[\mathrm{Gd}_{2} \mathrm{ac}\right]$. In any case, the absence of any $\mathrm{Gd}$ in the latter case would also indicate that isolated Gd(III) ions, present in the reaction mixture since the $\mathrm{Gd}_{2} \mathrm{O}_{3}$ white solids does disappear, are not efficiently coordinated to the MWCNT-ox. This in turn points at the hypothesis that species grafted on CNTs in the MWCNT- $\left[\mathrm{Gd}_{2} \mathrm{ac}\right]$ composite are likely similar to those in crystalline $\left[\mathrm{Gd}_{2} \mathrm{ac}\right]$.

Confirmation of this hypothesis was obtained through TGA, IR and Raman spectroscopies, elemental analysis, TEM observations and magnetization measurements. The supposed unreacted MWCNT-ox gives a very similar TGA trace as the original MWCNT-ox (Figure S2 and Table S1). TEM (Figure 1) shows that the functionalization process does not involve any further significant damage/modification to the MWCNT themselves, as expected and confirmed by the observation of the characteristic 002 reflection of graphitic layers in PXRD (Figure S3). TEM/EDX analysis of dense areas provides in addition the evidence of the presence of Gd only in MWCNT-[Gd $\left.\mathrm{G}_{2} \mathrm{ac}\right]$. The comparison of TEM images with different magnification (MWCNT-[Gd $\left.\mathrm{Gac}_{2}\right]$ vs. MWCNT-ox-un. in Figure 1) reveals the absence of any 
areas with a strong contrast, e.g. of Gd-rich aggregates or nanocrystals, which also indicates that the functionalization involves molecular Gd-containing species. This is confirmed by PXRD showing no diffraction peaks and therefore the absence of crystallites of [ $\left.\mathrm{Gd}_{2} \mathrm{ac}\right]{ }^{[21]}$ The spectroscopic data are not fully conclusive, but do support the functionalization. A weak band at $c a .2940 \mathrm{~cm}^{-1}$ appears in the IR spectrum of MWCNT-[Gd $\left.\mathrm{d}_{2} \mathrm{ac}\right]$ composite that can be ascribed to aliphatic C-H stretching, and thus to the methyl groups of acetate in $\left[\mathrm{Gd}_{2} \mathrm{ac}\right]$ (Figure S4 left). The very broad band covering the range $3000-3500 \mathrm{~cm}^{-1}$ likely corresponds to O-H stretching vibrations. Interestingly neither of these bands is present in the MWCNT-ox spectrum. There are also a few modifications of the $1000-2000 \mathrm{~cm}^{-1}$ range with respect to that of the MWCNT-ox that could be ascribed to the participation of surface carboxylic groups in coordination bonds as well as the presence of acetate moieties of $\left[\mathrm{Gd}_{2} \mathrm{ac}\right]$. These are the disappearance of a broad band around $1650 \mathrm{~cm}^{-1}$, the arising of a band at $c a .1730 \mathrm{~cm}^{-1}$ and the increased bands at 1515 and $1350 \mathrm{~cm}^{-1}$, corresponding to asymmetric and symmetric stretches of carboxylates (Figure S4 right). The Raman spectrum shows no trace of any vibrations from [ $\left.\mathrm{Gd}_{2} \mathrm{ac}\right]$, as it is usually the case for covalently-functionalized CNTs. The spectrum is thus characteristic of MWCNT, showing the defect (D) and graphitic (G) bands at $1354 \mathrm{~cm}^{-1}$ and $1593 \mathrm{~cm}^{-1}$ respectively (Figure S6). The ratio $I_{\mathrm{D}} / I_{\mathrm{G}}$, typically used to monitor the extent of covalent functionalization of CNT is 1.07, very similar to that found for MWCNT-ox, at 1.10, confirming again the process does not significantly damage the MWCNT core. Both D and G bands are however sharper in the case of the composite, being in addition shifted towards lower Raman shifts by respectively 3 and $12 \mathrm{~cm}^{-1}$. The $2 \mathrm{G}$ band found at $2933 \mathrm{~cm}^{-1}$ suffers a similar shift of $-15 \mathrm{~cm}^{-1}$, while the 2D band remains unchanged at $2712 \mathrm{~cm}^{-1}$. This shift of the $\mathrm{G}$ band may be taken as an indication of surface functionalization of the outer-wall of the MWCNT core. The Gd content determined by ICP amounts to $13.7 \%$ in mass $(0.87$ mmolGd per g), meaning that $c a .32 \%$ of the mass of the composite consists of $\left[\mathrm{Gd}_{2} \mathrm{ac}\right]$, considering the molar mass of the [ $\left.\mathrm{Gd}_{2} \mathrm{ac}\right]$ complex without its 
lattice water molecules. This estimation, based on the assumption that Gd is in the form of dinuclear molecules similar to $\left[\mathrm{Gd}_{2} \mathrm{ac}\right]$ is consistent with termogravimetric analysis. Indeed, the final residue above $520{ }^{\circ} \mathrm{C}$ consists in $\mathrm{Gd}_{2} \mathrm{O}_{3}$, as indicated by PXRD (Figure S7), and amounts to $14.8 \%$, which in turn gives $c a .0 .82 \mathrm{mmolGd}$ per g. In addition, the main weight loss occurs in two close stages, the first one coinciding with the oxidation of acetate to carbonate in $\left[\mathrm{Gd}_{2} \mathrm{ac}\right]$ (Figure S8). It is also important to note that the second stage involving the oxidation of the CNTs occurs at lower temperatures with respect to MWCNT-ox (Figure S2 and Table S1), likely due to the intimate presence of oxygen-rich Gd species.

Overall, a confident schematic representation of the composite material is thus that of MWCNT whose outer wall has been severely oxidized and intimately covered with molecular Gd-rich species, very likely similar to the $\left[\mathrm{Gd}_{2} \mathrm{ac}\right]$ molecule and strongly bound to the MWCNT either through carboxylic functions on the outer wall and on polyaromatic amorphous carbons and/or through strong multiple hydrogen bonds, as depicted in Scheme 1.

\subsection{Magnetic study}

The magnetic properties of $\left[\mathrm{Gd}_{2} \mathrm{ac}\right]$ were already reported in a previous work. ${ }^{[8]}$ Based on that, the temperature and field dependent magnetization of the composite material MWCNT[Gd $\mathrm{G}_{2} \mathrm{ac}$ ] provides an alternative evidence of the presence of $\mathrm{Gd}(\mathrm{III})$ ions, as well as their direct quantification. Note that, while the MWCNT-ox presents a magnetization per unit mass that remains almost constant from $c a$. $40 \mathrm{~K}$ down to $2 \mathrm{~K}$, MWCNT-[Gd $\mathrm{Gac}_{2}$ exhibits a continuous increase in the same temperature range (Figure 2, inset), reminiscent of a Curie law and ascribable to the Gd(III) ions it contains. Furthermore, while the magnetization vs. field at $3 \mathrm{~K}$ for MWCNT-ox already saturates at ca. $0.6 \mathrm{~T}$ displaying a very small value of $0.33 \mathrm{emu} / \mathrm{g}$, the composite data only approaches saturation above ca. $3 \mathrm{~T}$, reaching $31.3 \mathrm{emu} / \mathrm{g}$ at $5 \mathrm{~T}$ (Figure 2). We ascribe the comparatively very small magnetic signal of MWCNT-ox to remaining magnetic seeds, which are used in their synthesis and not fully removed by the 
acidic oxidation process. Note that, since the magnetic seeds are not resolved by TEM, they have to be present in extremely small amount.

After removing the contribution of the MWCNT-ox in the sample, the magnetization data for MWCNT-[Gd $\left.\mathrm{Gac}_{2}\right]$ provides a measure of the Gd(III) content, at $0.836 \mathrm{mmol} / \mathrm{g}$. This value, in excellent agreement with the elemental and thermogravimetric analyses, would correspond to ca. $33 \%$ of the mass of the composite being $\left[\mathrm{Gd}_{2} \mathrm{ac}\right]$ molecules. The assumption that the detected $\mathrm{Gd}(\mathrm{III})$ ions are in the form of $\left[\mathrm{Gd}_{2} \mathrm{ac}\right]$ molecules is further supported by the analysis of the heat capacity data (see below). Note though that comparing the magnetization data for MWCNT- $\left[\mathrm{Gd}_{2} \mathrm{ac}\right]$ with that for bulk-[Gd $\left.\mathrm{Gd}_{2} \mathrm{ac}\right]$ evidences a slight but sizeable difference in the field dependence (Figure 2), which we ascribe to a different coupling strength between $s_{\mathrm{Gd} 1}=$ $7 / 2$ and $s_{\mathrm{Gd} 2}=7 / 2$ spins within each molecular dimer. To provide a quantitative analysis, we fit the magnetization data to a model based on the isotropic exchange Hamiltonian $H=-$ $J\left(s_{\mathrm{Gd} 1} S_{\mathrm{Gd} 2}\right)$. In agreement with previous studies, ${ }^{[8]}$ bulk- $\left[\mathrm{Gd}_{2} \mathrm{ac}\right]$ is characterized by a weak ferromagnetic coupling $(J=0.07 \mathrm{~K})$. We find a weak coupling strength also for the $\left[\mathrm{Gd}_{2} \mathrm{ac}\right]$ molecules in the composite material, though the $s_{\mathrm{Gd} 1}-s_{\mathrm{Gd} 2}$ coupling is here antiferromagnetic with $J=-0.05 \mathrm{~K}$. Given the extreme sensitivity of the exchange coupling in carboxylicbridged pairs of Gd(III) ions to slight changes of the bridge structural parameters, ${ }^{[22]}$ it is not surprising that the $s_{\mathrm{Gd} 1}-S_{\mathrm{Gd} 2}$ coupling in the $\left[\mathrm{Gd}_{2} \mathrm{ac}\right]$ molecules changes its sign when going from bulk crystalline form to the grafting onto MWCNTs in the composite material. 


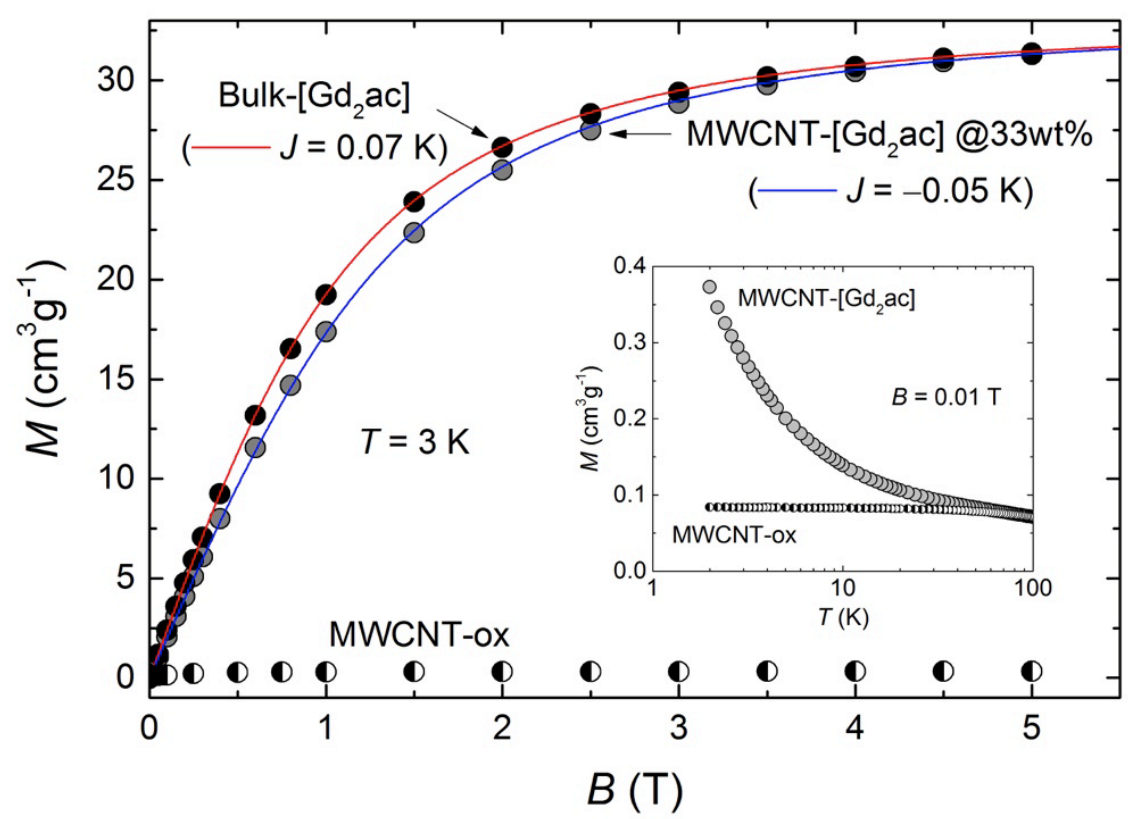

Figure 2. Field and temperature (inset) dependence of the mass magnetization of MWCNTox and the composite material MWCNT-[Gd $\left.\mathrm{G}_{2} \mathrm{ac}\right]$, respectively at $T=3 \mathrm{~K}$ and in a $d c$ field $B=$ $0.01 \mathrm{~T}$. Isothermal magnetization data for bulk-[Gd $\mathrm{Gac}_{2} \mathrm{are}$ also shown for comparison. Red

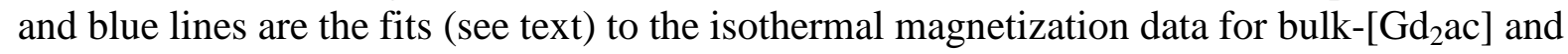
MWCNT- $\left[\mathrm{Gd}_{2} \mathrm{ac}\right]$ (after removing the contribution of the MWCNT-ox), respectively.

On basis of the gathered evidence, we will use in the following the mass formulation (MWCNT-ox) $)_{0.67}\left[\mathrm{Gd}_{2} \mathrm{ac}\right]_{0.33}$ for the composite, and thus derive the magnetothermal properties of its $\left[\mathrm{Gd}_{2} \mathrm{ac}\right]$ content after subtracting the MWCNT-ox contribution. We first turn to the determination of the magnetic entropy change $\Delta S_{\mathrm{m}}$ from molar magnetization data $M_{\mathrm{m}}(T, B)$, employing the Maxwell relation $\Delta S_{\mathrm{m}}(T)=\int\left[\partial M_{\mathrm{m}}(T, B) / \partial T\right] \mathrm{d} B$. Thus, from the magnetization data in Figure S9, $\Delta S_{\mathrm{m}}$ curves in the range 3-9 $\mathrm{K}$ are determined for applied field changes $\Delta B$ from 1 to 5 T. The values so-obtained (Figure S10), for example $-\Delta S_{\mathrm{m}}=24.7 \mathrm{~J} \mathrm{~kg}^{-1} \mathrm{~K}^{-1}$ at $T$ $=3 \mathrm{~K}$ for $\Delta B=3 \mathrm{~T}$, are the same as those for bulk-[Gd $\mathrm{Gac}_{2}$, confirming that the molecules grafted on the MWCNTs are similar to those in bulk-[Gd $2 \mathrm{ac}]$, and that they maintain intact their large MCE.

\subsection{Heat capacity study}


We measured the temperature dependence of the heat capacity, $c_{\mathrm{p}}$, of MWCNT-ox and the composite MWCNT-[Gd $\mathrm{Ga}_{2} \mathrm{ac}$, both in zero-field and in applied $d c$ fields $B=1,3$ and $7 \mathrm{~T}$. This allows the determination of the heat capacity of the $\left[\mathrm{Gd}_{2} \mathrm{ac}\right]$ component within the composite (Figure 3), and its comparison with that of bulk [ $\left.\mathrm{Gd}_{2} \mathrm{ac}\right]$ (Figure S11). At low temperature, $c_{\mathrm{p}}$ of MWCNT-ox depends on the applied magnetic field, though it roughly is two orders of magnitude smaller than $c_{\mathrm{p}}$ of MWCNT-[Gd $\left.\mathrm{d}_{2} \mathrm{ac}\right]$ for the same values of $T$ and $B$, in agreement with magnetization data (Figure 2). At high temperatures, non-magnetic contributions arising from thermal vibrations within the solids dominate, and not surprisingly, these are larger for the crystalline bulk-[Gd $\left.\mathrm{Gd}_{2} \mathrm{ac}\right]$ with respect to the amorphous MWCNT-[Gd $\left.\mathrm{d}_{2} \mathrm{ac}\right]$ composite, after subtracting the heat capacity of MWCNT-ox. We attribute the non-magnetic $c_{\mathrm{p}}$ of the [Gd $\left.\mathrm{d}_{2} \mathrm{ac}\right]$ component within the composite to optical phonons modes localized within the molecular units. The magnetic contribution to the heat capacity of the [ $\left.\mathrm{Gd}_{2} \mathrm{ac}\right]$ component within the composite is dominated by a Schottky-type anomaly arising from the splitting of the $s_{\mathrm{Gd} 1}$ and $S_{\mathrm{Gd} 2}$ pairs by the superexchange coupling and applied magnetic field, which shifts the anomaly towards higher temperatures on increasing the field value. A nice description of the experimental data is obtained by the Schottky curves (solid lines in Figure 3b) calculated for $J$ $=-0.05 \mathrm{~K}$, as obtained from the fit of the magnetization data, and the corresponding values of the applied magnetic field ( $B=1,3$ and 7 T). Interestingly, the onset of a magnetic phase transition, which is well evident in the zero-field heat capacity of bulk-[Gd $\mathrm{Gc}_{2}$ (Figure S11), ${ }^{[8]}$ is not present in $c_{\mathrm{p}}(T, 0)$ of $\left[\mathrm{Gd}_{2} \mathrm{ac}\right]$ molecules within the composite. This evidence demonstrates that the long-range magnetic order is suppressed in the composite. These differences aside, the data are very similar to bulk-[Gd $\mathrm{Gac}_{2} \mathrm{c}$. The values of the applied magnetic field that we employ are sufficiently high to decouple the individual spin centers, which are otherwise correlated by the relatively small superexchange interaction which characterizes the $s_{\mathrm{Gd} 1}$ and $s_{\mathrm{Gd} 2}$ pairs in each $\left[\mathrm{Gd}_{2} \mathrm{ac}\right]$ molecule, i.e., $J=0.07 \mathrm{~K}$ and $-0.05 \mathrm{~K}$ for the bulk crystalline form and the composite material, respectively. 

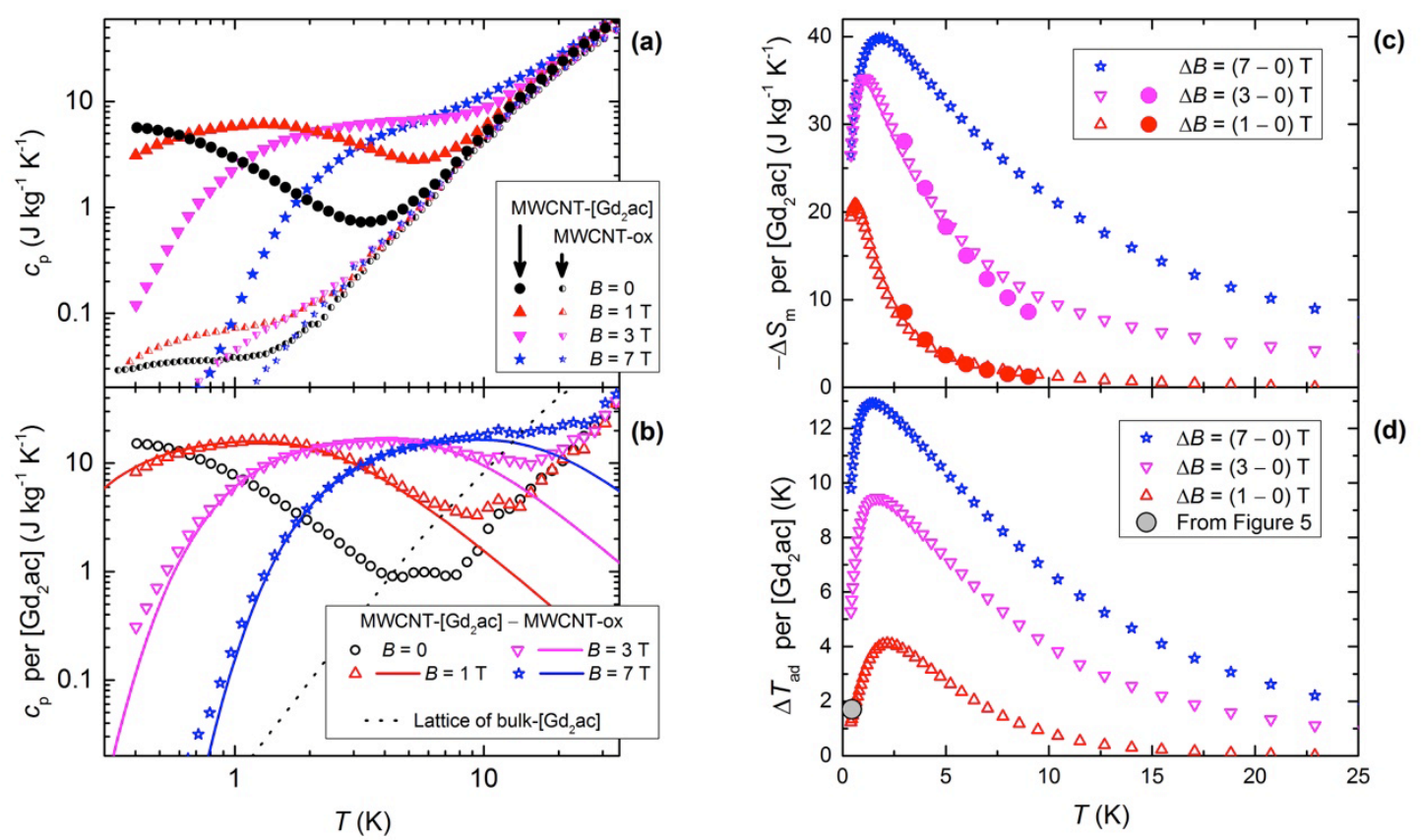

Figure 3. (a) Temperature-dependence of the mass heat capacity of MWCNT-ox and the composite material MWCNT-[Gd $\mathrm{G}_{2} \mathrm{ac}$ ], for selected applied field values, as labeled. (b) Temperature-dependence of the heat capacity of MWCNT-[Gd $\mathrm{Gac}_{2}$, after subtracting the MWCNT-ox contribution, for selected applied field values, as labeled. Solid lines are the Schottky heat capacities (see text), while dashed line is the lattice contribution to the heat capacity of bulk-[Gd $\mathrm{Gd}_{2} \mathrm{ac}$, shown for reference. (c) Temperature-dependence of the magnetic entropy change of the composite material at the indicated changes of the applied field, as obtained from heat capacity (empty symbols) and magnetization (filled symbols) data. (d) Temperature-dependence of the adiabatic temperature change of the composite material at the indicated changes of the applied field, as obtained from heat capacity (empty symbols) data and direct measurements of the MCE (see Figure 5).

Next, we derived the temperature-dependence of the magnetic entropy change $\Delta S_{\mathrm{m}}$ and adiabatic temperature change $\Delta T_{\text {ad }}$ from the experimental heat capacity, using known procedures. ${ }^{[6]}$ Figures 3c and 3d show the temperature-dependence of $\Delta S_{\mathrm{m}}$ and $\Delta T_{\mathrm{ad}}$, respectively, for selected values of the applied field change, $\Delta B=(1-0) \mathrm{T}$, (3-0) T and (7 $0)$ T. As can be seen, the so-calculated $\Delta S_{\mathrm{m}}(T, \square B)$ data nicely superimpose onto the equivalent set of $\Delta S_{\mathrm{m}}(T, \square B)$ data previously obtained from magnetization data. Besides, we note in Figure 3d that the so-calculated value of $\Delta T_{\mathrm{ad}}=1.9 \mathrm{~K}$ for $T=0.6 \mathrm{~K}$ and $\Delta B=(1-0) \mathrm{T}$ is corroborated by a direct experimental measurement of the MCE, which will be discussed 
below. Remarkably and expectedly, the MCE of the $\left[\mathrm{Gd}_{2} \mathrm{ac}\right]$ content in the composite material is significantly large, e.g., it reaches $-\Delta S_{\mathrm{m}}=40.0 \mathrm{~J} \mathrm{~kg}^{-1} \mathrm{~K}^{-1}$ at $T=1.8 \mathrm{~K}$ and $\Delta T_{\text {ad }}=12.8 \mathrm{~K}$ at $T=1.6 \mathrm{~K}$, both for $\Delta B=(7-0) \mathrm{T}$. More importantly, we evidence that, under the experimental conditions employed, the values we calculate for the MCE of the $\left[\mathrm{Gd}_{2} \mathrm{ac}\right]$ content in the composite material are about indistinguishable from the corresponding ones which were obtained for crystalline bulk-[Gd $2 \mathrm{ac}] .{ }^{[8]}$ Therefore, we can safely conclude that the $\left[\mathrm{Gd}_{2} \mathrm{ac}\right]$ molecules hold intact their functionality after being grafted to the MWCNTs.

\subsection{Thermal conductivity}

For studying the thermal transport, a longitudinal steady-state method providing very precise thermal conductance $(K)$ values was used. ${ }^{[23]}$ Consequently, bulk samples with well-defined dimensions were required in order to calculate the thermal conductivity, $\kappa$, as $\kappa=K \cdot L / A$, where $L$ and $A$ are the length and the cross-section of the sample, respectively (see Methods and S.I. for details on sample preparation and measuring method). Results of measurements are given in Figure 4, Figure S12 and Figure S13.

The data obtained in the range 4.5-155 $\mathrm{K}$ for a pressed pellet of bulk-[Gd $\left.\mathrm{Gac}_{2}\right]$ powder with a density, $\rho_{a p}$, of $1.80 \mathrm{gcm}^{-3}$ (empty circles in Figure 4) evidence the expected poor thermal conductivity of this molecular cooler, with values of $\kappa$ decreasing from $0.02 \mathrm{Wm}^{-1} \mathrm{~K}^{-1}$ at 153 $\mathrm{K}$ down to $3.2 \square \times \square 10^{4} \mathrm{Wm}^{-1} \mathrm{~K}^{-1}$ at $4.5 \mathrm{~K}$. The values in the high-temperature range are actually of the same order as those derived for single crystals of molecular coordination compounds, measured by relaxation microcalorimetry, ${ }^{[11]}$ and are a consequence of the bottleneck effect against heat transport caused by the weak bonds between molecules. At low temperatures, the values of $\kappa$ are about two orders of magnitude lower than those obtained in steady-state longitudinal-heat flow conditions for pressed powders of the gadolinium sulfate salt, $\mathrm{Gd}_{2}\left(\mathrm{SO}_{4}\right)_{3} \cdot 8 \mathrm{H}_{2} \mathrm{O},{ }^{[24]}$ i.e., the material used in early magnetic refrigeration studies. ${ }^{[25]}$ For 
comparison, crystals of GGG were reported to show thermal conductivities in the range of 10$200 \mathrm{Wm}^{-1} \mathrm{~K}^{-1}$ at temperatures in the range $2-30 \mathrm{~K},{ }^{[26]}$ also determined in steady-state conditions.

In the temperature range of interest for this study, i.e., below $10 \mathrm{~K}$, the $\kappa(T)$ data of the bulk$\left[\mathrm{Gd}_{2} \mathrm{ac}\right]$ pellet can be fitted to an expression of the form $\kappa_{\text {bulk }}=\square T^{n}$, with $\square=3.4 \square 10^{-5}$ $\mathrm{Wm}^{-1} \mathrm{~K}^{-\mathrm{n}-1}$ and $n=1.58$. The fact that the temperature-dependence of $\kappa \square \square$ is weaker than the $T^{3}$ expected for phonon contribution would suggest that the mean-free-path of phonons in this temperature-range is limited by scattering with crystal defects, such as grain boundaries and dislocations, likely present due to the polycrystalline nature of the material and the high pressure used to prepare the pellet, respectively. Another sample defect, the remaining macroporosity resulting from the rather inefficient densification of the $\left[\mathrm{Gd}_{2} \mathrm{ac}\right]$ powder (the apparent sample density is only ca. 85\% with respect to that of the crystal), may represent a further factor in the low $\kappa$ values derived. Even keeping in mind that the thermal conductivity of denser pressed powders or of single crystals can be expected to be higher, ${ }^{[27]}$ the first set of values of $\kappa$ reported here for a molecular cooling material are fairly small. This means that the use of molecular coolers such as $\left[\mathrm{Gd}_{2} \mathrm{ac}\right]$ in refrigerators would require very large $A / L$ ratios of the cooling object, i.e., very spread/thin objects to allow the required rapid heat flow. As will be intuited in the next section on basis of the direct measurements of the MCE, this could only be valid for local, on-chip refrigeration by thin layers of the molecular magnetic cooler. 


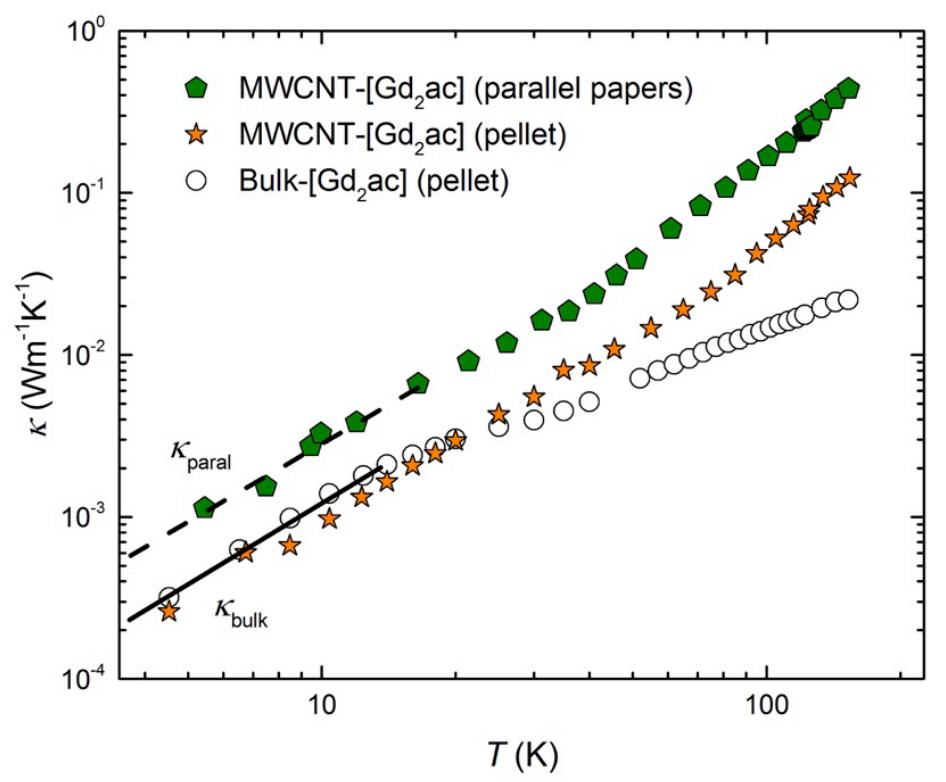

Figure 4. Temperature-dependence of the thermal conductivity of bulk-[Gd $\left.\mathrm{Gd}_{2} \mathrm{ac}\right]$ (empty black symbols) and MWCNT-[Gd $\mathrm{G}_{2} \mathrm{ac}$ (filled cyan symbols) pressed pellets and of an array of parallel buckypaper sheets of MWCNT-[Gd $\left.\mathrm{G}_{2} \mathrm{ac}\right]$ (filled red symbols). The full line is a fit of the bulk-[Gd $\left.\mathrm{G}_{2} \mathrm{ac}\right]$ data below $15 \mathrm{~K}$ to the expression $\kappa_{\text {bulk }}=\square \Gamma^{n}$, with $\square=3.4 \square \square 10^{5} \mathrm{Wm}^{-}$ ${ }^{1} \mathrm{~K}^{-\mathrm{n}-1}$ and $n=1.58$, while the dashed line is a fit of the parallel buckypaper sheets of MWCNT-[Gd $\left.\mathrm{Gac}_{2}\right]$ data to $\kappa_{\text {paral }}=\square \Gamma^{n}$, with $\square=7.9 \square 10^{-5} \mathrm{Wm}^{-1} \mathrm{~K}^{-\mathrm{n}-1}$ and $n=1.56$.

Thermal conductivity of the composite MWCNT-[Gd $\mathrm{Gd}_{2} \mathrm{ac}$ was first measured on a pressed pellet made from crushed pieces of buckypapers. The apparent mass density of this pellet was 1.6 times higher than that of a single MWCNT-[Gd $\left.\mathrm{d}_{2} \mathrm{ac}\right]$ buckypaper, estimated as $1.00 \mathrm{~g} / \mathrm{cm}^{3}$ (note that $33 \%$ of the mass is due to $\left[\mathrm{Gd}_{2} \mathrm{ac}\right]$ ). The obtained thermal conductivity values (Figure 6) for temperatures below $15 \mathrm{~K}$ are poor, indeed similar to those of $\left[\mathrm{Gd}_{2} \mathrm{ac}\right]$. At higher temperatures, though, $\kappa$ is significantly higher than that of $\left[\mathrm{Gd}_{2} \mathrm{ac}\right]$, for example $c a .0 .2 \mathrm{Wm}^{-}$ ${ }^{1} \mathrm{~K}^{-1}$ at $155 \mathrm{~K}$, i.e., about one order of magnitude of increase. Even at these temperatures, the thermal conductivity of the pellet is still low compared with that of aligned and densified MWCNT sheets $(1-2 \mathrm{~W} / \mathrm{m} \cdot \mathrm{K}$ at $155 \mathrm{~K}) .{ }^{[28]}$ While the low mass-density of the MWCNT papers, the lack of alignment and the interfacial resistance between CNTs within such papers typically greatly reduce $\kappa$, here additionally, the resulting arrangement upon pellet formation 
from pieces of buckypaper is likely not efficient, resulting in further thermal resistance at macroscopic contacts among these pieces to the detriment of the thermal conductivity. To allow a more representative measurement of the thermal conductivity of the MWCNT[Gd $\mathrm{Gac}_{2} \mathrm{ac}$ composite, we therefore made a sample of large pieces of buckypapers packed in a parallel disposition and embedded in a resin acting as mechanical support. This sample was cut and polished to assure that all buckypapers had the same length (that of the sample) and also to minimize the amount of embedding resin (Figures S12 and S13), whose volume fraction was estimated. Measurements were then performed parallel to the paper orientation, and corrected for the contribution of the embedding resin (see Experimental Section and Supporting Information). The resulting thermal conductivity of buckypapers of MWCNT[Gd $\mathrm{Gac}_{2}$ is shown in Figure 4. Qualitatively, the observed monotonous $\kappa$ increase with temperature is in accordance with previously reported experimental data. ${ }^{[29]}$ Interestingly, the MWCNT-[Gd $\left.\mathrm{d}_{2} \mathrm{ac}\right]$ buckypapers now exhibit a significant increase of $\kappa \square$ with respect to [Gd $\mathrm{d}_{2} \mathrm{ac}$ ] over the whole temperature range. Most importantly, $\kappa$ is increased by a factor of 2 to 3 below $20 \mathrm{~K}$, and the experimental data can be fitted to an expression of the form $\kappa_{\text {paral }}=\square \Gamma^{n}$, with $\square=7.9 \square \square 10^{-5} \mathrm{Wm}^{-1} \mathrm{~K}^{-\mathrm{n}-1}$ and $n=1.56$. These values are now comparable to those derived on vertically-aligned arrays of MWCNTs (determined using a longitudinal steadystate technique implemented in a physical property measurement system), ${ }^{[30]}$ even if the functionalization with $\left[\mathrm{Gd}_{2} \mathrm{ac}\right]$ molecules is expected to introduce further phonon scattering or/and interfacial resistance. ${ }^{[31]}$ Further analysis was not performed since it is difficult to find theoretical predictions for it due to the very diverse sources of thermal resistance (e.g., large variety of crystal defects, interlayer scattering processes, intertube thermal resistance). Eventually, considering that the MWCNTs are completely disordered within the buckypapers, significant improvements might be expected if some alignment could be induced. ${ }^{\text {[32] }}$ 


\subsection{Direct measurement of the magnetocaloric effect}

As concluded in previous sections, MWCNT-[Gd $\left.\mathrm{G}_{2} \mathrm{ac}\right]$ buckypapers contain $\left[\mathrm{Gd}_{2} \mathrm{ac}\right]$ molecules with full functionality and show higher thermal conductivity than a pressed pellet of bulk[Gd $\left.\mathrm{d}_{2} \mathrm{ac}\right]$ powder. In order to evaluate the cryogenic cooling performance, direct measurements of the magnetocaloric effect were carried out on both materials by following the temperature evolution induced by continuous field variations over magnetization-demagnetization cycles under controlled quasi-adiabatic conditions (cycles were carried out while holding constant the temperature of the bath which surrounds the platform holding the sample, see Supporting Information for details), as some of us previously described for other molecular nanomagnets. ${ }^{[33]}$ However, the scope of these experiments is twofold: in addition to assess the materials cooling performance, they provide the corroboration of the MCE results which were inferred from heat capacity and magnetization data. Even if the independent evaluations of the MCE using magnetization and heat capacity data, respectively, are coherent with each other, they are indirect measurements that rely on numerical integrations from which significant errors might be expected. ${ }^{[34]}$

We started with two different samples of bulk-[Gd $\left.\mathrm{Gac}_{2}\right]$ for our experiments, which we performed under the same conditions of bath temperature $\left(T_{0}=1.96 \mathrm{~K}\right)$ and applied magnetic field cycle $(0 \rightarrow 1 \mathrm{~T} \rightarrow 0)$. Both samples were prepared as pressed pellets in the form of cylinders, whose circular ends have a diameter of $3 \mathrm{~mm}$. The flat surface of a circular end lied on top of our sensor, i.e., the sample platform, while heat transport across this interface was improved by the presence of a thin layer of thermal grease for low temperatures. The difference between the two experiments resided in the mass of the samples, namely, $m=0.81$ mg and $8.1 \mathrm{mg}$, respectively. Therefore, other things being equal (e.g., diameter of the pressed pellets), each sample was characterized by a different height, which amounted to $h=0.06 \mathrm{~mm}$ and $0.6 \mathrm{~mm}$, respectively. With these experiments, as anticipated, we first sought the corroboration of the MCE results, which were indirectly inferred from heat capacity and 
magnetization data. Second, we wished to provide a close look at the thermalization of the molecule-based material by comparing how differently samples of drastically different heights behave in such measurements, that is, we should explore whether the intrinsic thermalization might have any influence on the resulting magnetic cooling.

Figure S14 and Figure S15 show how the temperature of bulk-[Gd $\left.\mathrm{Gac}_{2}\right]$ samples with $m=$ $0.81 \mathrm{mg}$ and $8.1 \mathrm{mg}$, respectively, evolved, while cycling the applied magnetic field $(0 \rightarrow 1 \mathrm{~T}$ $\rightarrow 0$ ). Note that the experimental values of the sample temperature are measured by a thermometer located on the platform holding the sample. In both cases, the sample gradually increased (decreased) its temperature on increasing (decreasing) the applied magnetic field, because of the magnetocaloric effect. Since the experiments were not carried out under ideal adiabatic conditions, the temperature change was a delicate balance between the MCE and the heat that was unavoidably released to (absorbed from) the thermal bath during the measurements. This heat transfer proceeded via the wires of known thermal conductance $\kappa_{w}(T)$, which held the sample platform. Qualitatively, this explains why, e.g., the sample with larger mass experimentally reached ca. $0.9 \mathrm{~K}$ at the end of the demagnetization process (Figure 5 and Figure S15), while the lowest temperature attained by the sample with the significantly smaller mass was ca. $1.5 \mathrm{~K}$ solely (Figure 5 and Figure S14). Actually in the latter case, the heat absorbed from the thermal bath prevailed over the MCE already prior to reach zero applied field (at the end of the demagnetization process, the temperature of the sample was ca. $1.9 \mathrm{~K}$, i.e., it nearly recovered thermal equilibrium with the bath). Consequently, these MCE experiments are not directly comparable to each other due to the remarkable influence of the sample mass and height, and a simulation was performed to explain the observed behavior and estimate temperature differences in ideal adiabatic conditions. 


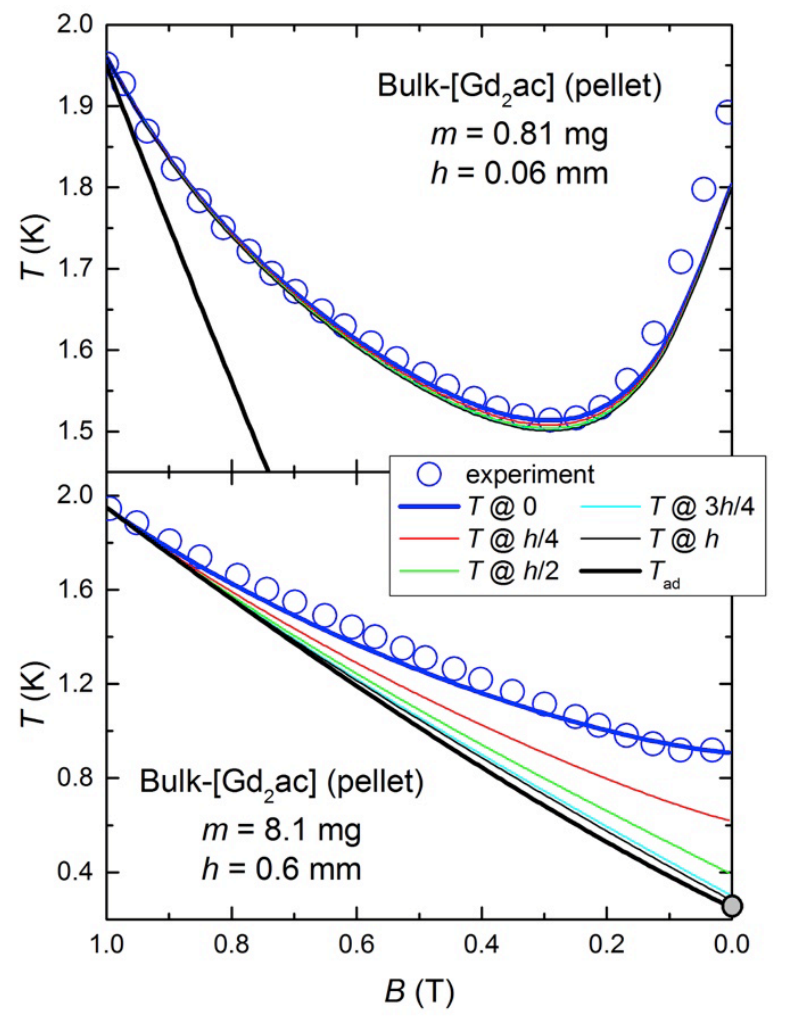

Figure 5. For a pellet sample of bulk-[Gd $\left.\mathrm{Gd}_{2} \mathrm{ac}\right]$ with height $h=0.06 \mathrm{~mm}$ (top panel) or $h=0.6$ $\mathrm{mm}$ (bottom panel), experimental sample temperature as a function of the applied field on demagnetization, from $T_{0}=1.96 \mathrm{~K}$ and $B_{0}=1 \mathrm{~T}$ initial conditions. The experimental values of the sample temperature are measured by a thermometer located on the platform holding the sample. For modeling the effect of the intrinsic thermal conductivity along $z$, we divide the height $h$ of the sample in 32 equidistant sections, and we calculate the temperature for each of them (see Supporting Information for details). Thin colored lines are representative temperatures calculated for $z=0, h / 4, h / 2,3 h / 4$ and $h$. Thick black line is the calculated temperature assuming this same measurement under adiabatic conditions.

From Figures S14 and S15 we extracted the experimental temperatures on demagnetization (1 $\mathrm{T} \rightarrow 0$ ), which are plotted as a function of the applied magnetic field in Figure 5, for both samples considered, together with the results of our simulation (see Supporting Information for details). Our hypothesis was that the temperature is not necessarily homogeneous across the whole sample, viz., we specifically assumed that there exists a temperature gradient which depends on the distance $z$ from the platform and, therefore, on the thermal conductivity of the sample. On basis of the experimental thermal conductivity of bulk- $\left[\mathrm{Gd}_{2} \mathrm{ac}\right]$ ( $\kappa_{\text {bulk }}$, see previous 
section), we calculated the temperatures corresponding to several distances from the platform. Thin solid lines in Figure 5 show the so-obtained representative temperatures for $z=0, h / 4$, $h / 2,3 h / 4$ and $h$, where the height $h$ of the sample either amounts to $0.06 \mathrm{~mm}$ or $0.6 \mathrm{~mm}$, respectively, depending on the sample considered. In the case of the sample with $h=0.06 \mathrm{~mm}$, it turns out that all calculated temperatures nearly collapse onto one curve only, which nicely reproduces the experimental data, as can be seen in Figure 5 (top panel). Not surprisingly, such a small thickness permits attaining a temperature that is almost instantly homogeneous across the entire sample. Differently, in the case of the sample with $h=0.6 \mathrm{~mm}$, a sizeable temperature gradient sets in inside the sample. As can be seen in Figure 5 (bottom panel), the temperatures calculated for $z=0$ describe very satisfactorily the experimental data. However, relatively lower temperatures are foreseen to occur in the sample, namely, the lower the temperature, the higher is the distance from the platform. This is understood since the heat that is transferred from the thermal bath $\left(T_{0}=1.96 \mathrm{~K}\right)$ enters into the sample from its bottom, which is in thermal contact with the platform. Therefore, a height of $0.6 \mathrm{~mm}$ (combined with a diameter of $3 \mathrm{~mm}$ ) is already sufficient for observing microscopically the competition between the heat released from the thermal bath and the magnetocaloric effect of the sample. By making use of the same model (see Supporting Information), we also calculate the adiabatic temperature $T_{\text {ad }}$ (thick line in Figure 5), that is, the temperature of the sample if there were no heat transferred from the thermal bath (i.e., under adiabatic conditions), which has therefore to be equal for both pellets. Note in our experiments that the larger the mass of the sample, the smaller is the influence of the hotter thermal bath, thus the closer is the behavior to the ideally adiabatic one, as expected. The marked dot in Figure 5 corresponds to $T_{\mathrm{ad}} \cong 0.28$ $\mathrm{K}$ reached at the end of the demagnetization, i.e., it provides the adiabatic temperature change $\Delta T_{\mathrm{ad}} \cong T_{0} \square 0.28 \mathrm{~K} \cong 1.68 \mathrm{~K}$ which is attained under the experimental conditions of $\Delta B=(1-$ 0) $\mathrm{T}$ and starting temperature $T_{0}=1.96 \mathrm{~K}$ or, equivalently, final temperature $T \cong 0.28 \mathrm{~K}$. Note 
in Figure 3 that the so-obtained value of $\Delta T_{\text {ad }}$ nicely corroborates the estimate that we independently obtained from the heat capacity data.

The larger is the mass of the sample, the smaller is the overall influence of the heat gained from the environment on demagnetization, i.e., the lack of adiabatic conditions. However, as the mass of the sample increases, so does its thickness, and the sample end in contact with the sensor (platform) experiences a lower cooling on demagnetization than the opposite end, presumably due to the poor thermal conductivity of the sample. Our model helps on disclosing further the role played by the thermal conductivity in our demagnetization experiments. Let us suppose that we can hypothetically boost the thermal conductivity of bulk-[Gd $\left.{ }_{2} \mathrm{ac}\right]$. For the sample with $h=0.6 \mathrm{~mm}$, Figure 6 shows how measurements under the same experimental conditions should evolve, depending on $\kappa$ that we vary in our simulations from $\kappa_{\text {bulk }}$ (as already considered for modeling the experimental data in the bottom panel of Figure 5) up to a ten-times larger thermal conductivity. Note that the solid lines shown in Figure 6 (top panel) correspond to the temperatures calculated at the bottom of the sample, i.e., the temperatures which are measured in the experiments. As nicely shown, the larger $\kappa$, the lower is the base temperature attained at the end of the demagnetization. For instance for $\kappa=$ $a \times \kappa_{\text {bulk, }}$, where $a=1.5,3,5$ and 10 , we obtain the base temperature $T \cong 0.8,0.7,0.6$ and $0.5 \mathrm{~K}$, respectively (Figure 6). Experimentally, we enhanced $\kappa$ by grafting the $\left[\mathrm{Gd}_{2} \mathrm{ac}\right]$ molecules to MWCNTs, and by further arranging buckypapers of this composite material in a parallel manner with respect to the heat flow. By so doing, the low-temperature thermal conductivity increased by a factor of 2 to 3 , up to $\kappa_{\text {paral }}$ (Figure 4). Therefore, direct measurements of the MCE in MWCNT- $\left[\mathrm{Gd}_{2} \mathrm{ac}\right]$ were carried out for a sample consisting in an array of a few dozens of parallel buckypapers mounted perpendicularly on top of the sensor platform. A thin layer of thermal grease facilitated heat conduction through the interface between sample and platform, while polytetrafluoroethylene wire was employed to hold the buckypapers together. 
Each buckypaper was cut as such to have a height of $c a .6 \mathrm{~mm}$. The sample contains $\left[\mathrm{Gd}_{2} \mathrm{ac}\right]$ molecules with a cumulative amount of $m_{\text {[Gd2ac] }}=3.3 \mathrm{mg}$. From the whole magnetizationdemagnetization cycle (Figure S16), we have extracted the experimental temperatures on demagnetization $\left(1 \mathrm{~T} \rightarrow 0\right.$, from $T_{0}=1.96 \mathrm{~K}$ ) as a function of the applied magnetic field in Figure 6 (bottom panel). Although the different sample geometry and different amount of the magnetic content impedes a direct comparison with previous results inferred from the bulk equivalent material, we note that the field-dependence of this sample temperature roughly is in between that of the two bulk-[Gd $\mathrm{Gac}_{2} \mathrm{ac}$ samples. As above, we can take advantage of our model (see above and Supporting Information for details) to interpret the experimental behavior. A nice description is found (thick solid line in Figure 6, bottom panel) by using as input into our model the thermal conductivity $\kappa_{\text {paral, }}$, which we measured for parallel buckypapers of MWCNT-[Gd $\left.{ }_{2} \mathrm{ac}\right]$ (see Figure 4). Importantly and as expected, the enhanced $\kappa_{\text {paral }}$ favors attaining respectively much lower temperatures, as clearly evident by repeating this calculation with the same input parameters but worse bulk thermal conductivity $\kappa_{\text {bulk }}$ (Figure 6, bottom panel). If we were in the hypothetical condition of increasing the thermal conductivity even further above $\kappa_{\text {paral }}$, then much lower temperatures should be attained, as anticipated (Figure 6). 


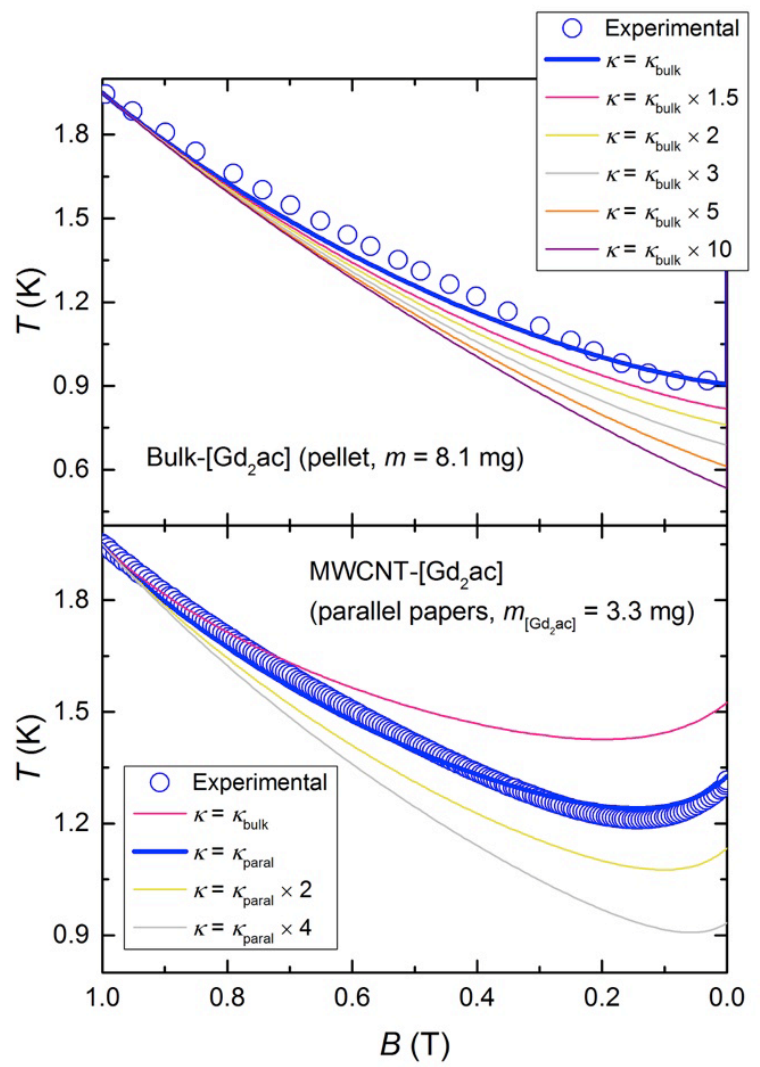

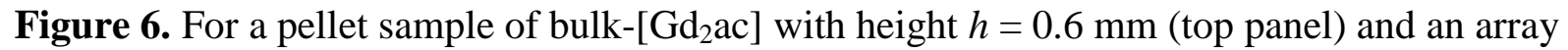
of parallel buckypapers of MWCNT-[ $\left.\mathrm{Gd}_{2} \mathrm{ac}\right]$ with $h=6.0 \mathrm{~mm}$ (bottom panel), experimental sample temperature as a function of the applied field on demagnetization, from $T_{0}=1.96 \mathrm{~K}$ and $B_{0}=1 \mathrm{~T}$ initial conditions. Solid lines are the temperatures calculated at $z=0$ (see main text) for different values of the intrinsic thermal conductivity $\kappa$ of the sample, as labeled.

The aforementioned analysis, including the comparison with the estimated behavior under adiabatic conditions, puts into evidence the following important aspects. For thin layers of molecular magnetic coolers, such as $h=0.06 \mathrm{~mm}$ (Figure 5, top panel), the experimental thermal conductivity of bulk-[Gd $\left.\mathrm{G}_{2} \mathrm{ac}\right]$ is already sufficient for thermalizing satisfactorily the sample within the time needed for the demagnetization, although heat gained from the environment can be highly detrimental. Therefore, micro-refrigeration using thin layers of molecular magnetic coolers seems a viable application for local, on-chip cooling, ${ }^{[14]}$ provided that the process is carried out under adiabatic conditions. Increasing the mass of the refrigerant magnetic material by using thicker layers provides a better cooling reservoir, 
although temperature gradients set in because of the poor thermal conductivity of these materials. Enhancing the thermal conductivity of molecular magnetic coolers, as in the MWCNT- $\left[\mathrm{Gd}_{2} \mathrm{ac}\right]$ composite material described here, is highly beneficial for applications using bulk samples, since it would bring significant improvements (Figure 6). A larger thermal conductivity reduces temperature gradients, which ultimately favors attaining lower temperatures.

\section{Conclusion}

The efficient attachment of carboxylate-bridged Gd(III) molecules onto oxidized multi-walled carbon nanotubes is reported as a facile strategy to fabricate hybrid composite molecular magnetic coolers with improved thermal conductivity. The magneto-thermal properties of the grafted molecules are shown to be similar to those of the parent bulk analogue, $\left[\mathrm{Gd}_{2}(\mathrm{OAc})_{6}\left(\mathrm{H}_{2} \mathrm{O}\right)_{4}\right] \cdot 4 \mathrm{H}_{2} \mathrm{O}$, one of the archetypal molecular magnetic coolers. In particular, its remarkably large magnetocaloric effect is maintained, while the magnetic order observed at ca. $0.2 \mathrm{~K}$ in the bulk material, is cancelled in the composite hybrid material, thus enabling cooling to even lower temperatures. The thermal conductivity of the bulk material is derived from a steady-state method, the very first report on the thermal conductivity of a molecular magnetic cooler at cryogenic temperatures, with relatively low values confirming it to be the main disadvantage of these materials. The thermal conductivity of the hybrid molecular cooler - carbon nanotube composite is shown to significantly surpass that of the bulk molecular magnetic cooler, by a factor of 2 to 3 in the temperature range of interest. Direct measurements of the MCE performed on samples of different thicknesses of bulk$\left[\mathrm{Gd}_{2}(\mathrm{OAc})_{6}\left(\mathrm{H}_{2} \mathrm{O}\right)_{4}\right] \cdot 4 \mathrm{H}_{2} \mathrm{O}$ corroborate the indirect determinations done on basis of magnetic and calorimetric data, and evidence two main facts: i) thinner samples or layers are not affected by adverse effects of the material poor thermal conductivity, while thicker ones present thermal gradients and reduced cooling performance at the contact surface; ii) the 
performance of thinner samples or layers is easily influenced by heat interchanges with the surroundings, while thicker ones act as better cooling reservoirs. These facts indicate that local micro-refrigeration with thin films of molecular magnetic coolers is a viable application in case of adiabatic conditions. For bulk refrigeration, the improved thermal conductivity of the composite is shown to result in a better performance since it allows attaining lower temperatures at the contact surface. Such improved performance opens the way towards applicable bulk molecular-based hybrid magnetic refrigerants.

\section{Experimental Section}

Materials synthesis.

MWCNT were purchased from Aldrich and used without further purification. Crystals of [ $\left.\mathrm{Gd}_{2}\left(\mathrm{CH}_{3} \mathrm{COO}\right)_{6}\left(\mathrm{H}_{2} \mathrm{O}\right)_{4}\right] \cdot 4 \mathrm{H}_{2} \mathrm{O}$ were prepared as previously described by warming $\mathrm{Gd}_{2} \mathrm{O}_{3}$ in a 2M aqueous solution of acetic acid. ${ }^{[8]}$ Purity of the crystals was checked by IR spectroscopy and powder X-ray diffraction. Determination of the C, H, O and Gd content was done by Mikroanalytisches Labor Pascher, Remagen, Germany.

Oxidized MWCNT (MWCNT-ox). These were obtained following similar conditions as previously reported. ${ }^{[35]}$ Specifically, a mixture of $20 \mathrm{~mL}$ sulfuric acid and $10 \mathrm{~mL}$ nitric acid was added to $1.51 \mathrm{~g}$ of raw MWCNT placed in a round-bottom flask equipped with a condenser. The heterogeneous mixture was warmed under vigorous stirring and kept for 30 min at $115-120{ }^{\circ} \mathrm{C}$ under reflux. After cooling to room temperature, $300 \mathrm{~mL}$ deionized water was then added resulting in a black dispersion that was filtered over a $0.22 \mu \mathrm{m}$ mixed cellulose esters membrane (GSWP, Millipore). The black solid recovered was washed thoroughly with deionized water until the filtrate was colorless. The wet black solid was directly dispersed in $500 \mathrm{~mL}$ deionized water resulting in a homogeneous stable solution. No significant aggregates were observed by optical microscopy, even after several months. The concentration of this stock MWCNT-ox solution was determined to be of 2.7(1) g/L through 
dry extracts and the weights of buckypapers formed by filtration of varying volumes of the mother solution on $0.22 \mu \mathrm{m}$ hydrophilic PVDF membranes (GVWP, Millipore). This stock MWCNT-ox solution was used in the following as prepared, only submitting the used volume to 2 min bath ultrasonication prior to use.

MWCNT- $\left[\mathrm{Gd}_{2} a c\right]$ composite. The targeted material was obtained by dissolving $0.235 \mathrm{~g}$ of $\left[\mathrm{Gd}_{2}\left(\mathrm{CH}_{3} \mathrm{COO}\right)_{6}\left(\mathrm{H}_{2} \mathrm{O}\right)_{4}\right] \cdot 4 \mathrm{H}_{2} \mathrm{O}$ in $10 \mathrm{~mL}$ deionized water and adding the resulting clear solution to $20 \mathrm{~mL}$ of the MWCNT-ox stock solution, then warming the initially homogeneous black mixture under stirring. The mixture rapidly became heterogeneous as a black solid deposited. The dispersion was kept at $90^{\circ} \mathrm{C}$ for 3 hours under vigorous stirring. After cooling to room temperature, the black solid was recovered by filtration over a $0.22 \mu$ m hydrophilic PVDF membrane and washed thoroughly with deionized water. The same process was repeated several times on various scales (up to 10 times, i.e. $2.4 \mathrm{~g}$ of $\left[\mathrm{Gd}_{2}\left(\mathrm{CH}_{3} \mathrm{COO}\right)_{6}\left(\mathrm{H}_{2} \mathrm{O}\right)_{4}\right] \cdot 4 \mathrm{H}_{2} \mathrm{O}$ and $200 \mathrm{~mL}$ of the MWCNT-ox stock solution) and did not produce materials with significantly different composition. The resulting buckypapers were used for all characterizations. The black solid can only be re-dispersed in water at very low concentration and the dispersion is actually not stable for long periods, deposition occurring within less than an hour. Elemental analysis (triplicate for $\mathrm{C}$ and $\mathrm{H}$, duplicate for $\mathrm{O}$ and $\mathrm{Gd}$ ) of the black buckypaper gave the following mass fractions: C, $61.60 \%$ H, $1.56 \%$; O, $22.7 \%$; Gd, $13.7 \%$.

Alternatively, a white slurry of $0.490 \mathrm{~g} \mathrm{Gd}_{2} \mathrm{O}_{3}$ dispersed in $20 \mathrm{~mL}$ of a $2 \mathrm{M}$ acetic acid aqueous solution was added to $40 \mathrm{~mL}$ of the MWCNT-ox stock solution. The mixture was also warmed under stirring to $90{ }^{\circ} \mathrm{C}$ and kept at that temperature for 3 hours, without any apparent modification, i.e. almost no solid deposited and the mixture remained black and homogeneous. The MWCNT content of this stable dispersion was recovered by filtration over a $0.22 \mu \mathrm{m}$ hydrophilic PVDF membrane and washed thoroughly with deionized water. As 
opposed to the material obtained by reaction with pre-formed $\left[\mathrm{Gd}_{2}\left(\mathrm{CH}_{3} \mathrm{COO}\right)_{6}\left(\mathrm{H}_{2} \mathrm{O}\right)_{4}\right] \cdot 4 \mathrm{H}_{2} \mathrm{O}$, this black solid was easily re-dispersed in deionized water, and proved to be unmodified MWCNT-ox.

Physical characterization

Infra-red spectra were acquired on neat samples using a Perkin Elmer Spectrum 100 apparatus equipped with an ATR device.

Raman spectra were obtained with a DILOR XY spectrometer equipped with a liquid $\mathrm{N}_{2^{-}}$ cooled CCD detector. Scattered light was collected through an X50 microscope objective lens of an Olympus BH-MA-2 microscope. The Si band at $520 \mathrm{~cm}^{-1}$ was used for frequency calibration. The $496.5 \mathrm{~nm}$ line of an $\mathrm{Ar}^{+}$laser was used as the excitation source, operating with $20 \mathrm{~mW}$ power, corresponding to $c a .1 \mathrm{~mW}$ power at the sample surface.

Thermogravimetric analysis (TGA) was performed with a TA Q5000 thermoanalyzer at $10^{\circ} \mathrm{C} /$ min under synthetic air at the Servicio de análisistérmico y calorimetría del Instituto de Ciencia de Materiales de Aragón (ICMA).

Powder X-ray diffraction (XRD) patterns were recorded through the X-Ray diffraction and fluorescence unit of the Servicio General de Apoyo a la Investigación-SAI, Universidad de Zaragoza, using a D-Max Rigakudiffractometer equipped with a Cu rotating anode and graphite monochromator to select the $\mathrm{Cu} \mathrm{K} \alpha_{1,2}$ wavelength.

Transmission electron microscopy (TEM) observations were done with a JEOL 2000 FXII instrument working at an acceleration voltage of $200 \mathrm{kV}$, at the Materials Electron Microscopy unit of the Servicio General de Apoyo a la Investigación-SAI, Universidad de Zaragoza. Drops of either the aqueous stable MWCNT-ox stock solution, an aqueous stable re-dispersion of the MWCNT content recovered after reaction with $\mathrm{Gd}_{2} \mathrm{O}_{3}$ and acetic acid and a dispersion of the MWCNT-[Gd $\left.\mathrm{G}_{2} \mathrm{ac}\right]$ composite were put onto copper grids with a carbon membrane film. 
Magnetic measurements were performed using a commercial SQUID magnetometer of the Physical Measurements unit of the Servicio General de Apoyo a la Investigación-SAI, Universidad de Zaragoza. The data were corrected for the experimentally measured contribution of the sample holder.

Heat capacities in the range $0.35-30 \mathrm{~K}$ were obtained using the relaxation method in a commercial ${ }^{3} \mathrm{He}$ set-up equipped with a $9 \mathrm{~T}$ magnet, at the same facility. Apiezon- $\mathrm{N}$ grease was used to provide good internal thermal contact between the heater, thermometer and sample, in the form of a pellet made from either the $\left[\mathrm{Gd}_{2}\left(\mathrm{CH}_{3} \mathrm{COO}\right)_{6}\left(\mathrm{H}_{2} \mathrm{O}\right)_{4}\right] \cdot 4 \mathrm{H}_{2} \mathrm{O}$ polycrystalline powder or buckypapers of MWCNT-ox and MWCNT-[Gd $\left.\mathrm{d}_{2} \mathrm{ac}\right]$ broken/crushed into small pieces.

Thermal conductivity was measured using a commercial adiabatic setup from Termis Ltd. provided with a thermal conductivity cell that determines the thermal conductance $(K)$ by means of a longitudinal steady-state method. In this setup, the heat interchange between the sample and surroundings is minimized by the use of vacuum conditions and a radiation shield located between the heat source and sink of the thermal conductivity cell, thus surrounding the lateral area of the sample. The measuring process consists of the establishment of a temperature difference between the ends of a sample with a well-defined cylindrical or prismatic geometry and the measurement of the heat power required to maintain such a temperature difference in stationary conditions. For each $K$ determination, this process is repeated twice, using a higher temperature increment in the second step. The thermal conductance is then calculated from the ratio of the additional heat power provided and the additional temperature difference reached during the second step. This differential method allows discarding constant or small spurious contributions to $K$. The thermal conductivity $\kappa$ can then be derived from the expression $\kappa=K \cdot L / A$, where $L$ and $A$ are the length and the cross-section of the sample, respectively. Further details of the setup, measuring process, and data treatment have been described previously. ${ }^{[23]}$ 
The two first samples for thermal conductivity measurement were made of finely powdered polycrystalline $\left[\mathrm{Gd}_{2}\left(\mathrm{CH}_{3} \mathrm{COO}\right)_{6}\left(\mathrm{H}_{2} \mathrm{O}\right)_{4}\right] \cdot 4 \mathrm{H}_{2} \mathrm{O}$ and of buckypapers of MWCNT-[Gd $\left.\mathrm{Gac}_{2}\right]$ composite broken/crushed into small pieces, respectively, and pressed into discs of $c a .1 .3 \mathrm{~cm}$ diameter under a pressure of $c a .820 \mathrm{MPa}\left(12\right.$ tons on a surface of $1.410^{-4} \mathrm{~m}^{2}$ ). Further, a third sample was prepared as follows. Buckypapers of the MWCNT-[Gd $\left.\mathrm{ac}_{2}\right]$ composite were cut into rectangular pieces that were then fixed together in a compact and parallel arrangement within LR White acrylic embedding resin for Electron Microscopy (see Figure S15 for images of the process). The resin is aimed to provide mechanical support to the buckypapers, which must be pressed between the heat sink and source of the thermal conductivity cell for good thermal contact. The sample was first polished to assure that all buckypapers had the same length (that of the sample), and then the excess LR White resin surrounding the parallel pieces of buckypaper was cut out. Optical microscopy was used on this sample to measure the final surface fraction of buckypapers (80.1\%), and also to estimate the dimensions of up to 7 pieces of previously weighted buckypaper, in order to obtain its apparent density $\left(1.00 \mathrm{~g} / \mathrm{cm}^{3}\right)$. Eventually, a fourth sample of similar height than the third was prepared of pure LR White resin, whose apparent density was also determined $\left(1.12 \mathrm{~g} / \mathrm{cm}^{3}\right)$. All the dimensions, weights and derived apparent densities of the measured samples are collected in Table S2.

In order to calculate the thermal conductivity of the buckypapers from those of the third and fourth samples, the volume fraction of each component of the buckypaper / resin sample must be known. It was first considered that the buckypapers were not internally embedded with resin, i.e., that the volume fraction equals the surface fraction obtained by optical microscopy. However, this supposition was discarded, since it was incompatible with the apparent density of the sample $\left(1.32 \mathrm{~g} / \mathrm{cm}^{3}\right)$. Thus, the mass and volumes of each component were calculated using the total sample mass, the surface fraction and the apparent density of the buckypapers and resin. As a result, calculation indicates that a resin volume fraction of 0.27 is located 
inside the buckypapers. To assess this result, the void volume fraction within the buckypapers was roughly estimated using apparent densities of 2.71 and $1.35 \mathrm{~g} / \mathrm{cm}^{3}$ for $\left[\mathrm{Gd}_{2} \mathrm{ac}\right.$ ] molecules and MWCNTs, respectively, resulting in a $70 \%$ of the void volume occupied by the resin. The thermal conductivity of the buckypapers was finally calculated using the rule of mixtures with a resin volume fraction of 0.46 and the $\kappa$ data obtained for the pure LR White sample (Figure S14).

Direct MCE measurements were performed using the same set-up as for the heat capacity measurements. Cylindrical pressed pellet samples of $\left[\mathrm{Gd}_{2}\left(\mathrm{CH}_{3} \mathrm{COO}\right)_{6}\left(\mathrm{H}_{2} \mathrm{O}\right)_{4}\right] \cdot 4 \mathrm{H}_{2} \mathrm{O}$ polycrystalline powder were mounted on a sapphire plate (platform) with their axes perpendicular to the plate. The platform is attached to a Cernox resistance thermometer, and also by wires to a controlled thermal bath. For the MWCNT-[Gd $\left.\mathrm{ac}_{2}\right]$ composite, an array of $6 \times 3 \mathrm{~mm}^{2}$ pieces of buckypapers packed and held parallel to each other by a PTFE wire was used and mounted with the buckypapers perpendicular to the sapphire plate. Each MCE measurement started with the sample at zero applied magnetic field and at temperature $T_{0}$, and comprised: (a) gradual application of a magnetic field, up to a maximum $B_{0}$; (b) relaxation until the sample reached the thermal equilibrium with the bath; (c) gradual demagnetization down to $B_{0}=0$; and (d) relaxation at zero field until the sample reached thermal equilibrium at $T_{0}$. During the whole procedure, the temperature $T$ and applied magnetic field $B_{0}$ were recorded continuously. More details of the experimental conditions and data treatment are provided in the supporting information.

\section{Supporting Information}

Supporting Information is available from the Wiley Online Library or from the author.

\section{Acknowledgements}

Authors acknowledge funding from the Spanish MINECO through projects MAT2014-53961R (O. R., E. N., G. L.), MAT2015-70868-ERC (O. R.), MAT2015-68204-R (M. E., G. L.) as well as for a postdoctoral contract (to G. L.). The authors are grateful to Dr. M. L. Sanjuan (ICMA) for the acquisition of Raman spectra. 
Received: ((will be filled in by the editorial staff))

Revised: ((will be filled in by the editorial staff))

Published online: ((will be filled in by the editorial staff))

\section{References}

[1] a) W. F. Giauque, J. Am. Chem. Soc.1927, 49, 1864; b) R. P. Hudson, Principles and Applications of Magnetic Cooling, North-Holland, Amsterdam 1972; c) V. K. Pecharsky, K.

A. Gschneider, J. Magn. Magn. Mater.1999, 25, 44

[2] a) A. T. Serlemitsos, B. A. Warner, S. Castles, S. R. Breon, Adv. Cryogenics1990, 35B, 1431; b) P. J. Shirron, Cryogenics 2014, 62, 130

[3] A. M. Tishin, Y. I. Spichkin, The magnetocaloric effect and its applications (Series in Condensed Matter Physics), IOP, Bristol 2003

[4] J. A. Barclay, W. A. Steyert, Cryogenics1982, 22, 73

[5] Electronic schemes currently exploited produce only limited $\square T$ of the order of $0.1 \mathrm{~K}$, see

J. T. Muhonen, M. Meschke, J. P. Pekola, Rep. Prog. Phys.2012, 75, 046501

[6] M. Evangelisti, E. K. Brechin, Dalton Trans.2010, 39, 4672

[7] J. W. Sharples, D. Collison, Polyhedron2013, 54, 91

[8] M. Evangelisti, O. Roubeau, E. Palacios, A. Camón, T. N. Hooper, E. K. Brechin, J. J. Alonso, Angew. Chem. Int. Ed.2011, 50, 6606

[9] a) G. Lorusso, J. W. Sharples, E. Palacios, O. Roubeau, E. K. Brechin, R. Sessoli, A.

Rossin, F. Tuna, E. J. L. McInnes, D. Collison, M. Evangelisti, Adv. Mater.2013, 25, 4653; b)

G. Lorusso, M. Palacios, G. S. Nichol, E. K. Brechin, O. Roubeau, M. Evangelisti, Chem.

Commun.2012, 48, 7592; c) R. Sibille, T. Mazet, B. Malaman, M. Francois, Chem. Eur.

$J .2012,18,12970$

[10] M. J. Martínez-Pérez, O. Montero, M. Evangelisti, F. Luis, J. Sesé, S. Cardona-Serra, E. Coronado, Adv. Mater.2012, 24, 4301 
[11] Thermal conductivity measurements of molecular coordination compounds are extremely scarce, but values reported are quite low, see R. Jakobi, H. Romstedt, H. Spiering, P. Gütlich, Angew. Chem. Int. Ed. Engl.1992, 31, 178

[12] B. L. Huang, Z. Ni, A. Millward, A. J. H. McGaughey, C. Uher, M. Kaviani, O. Yaghi, Int. J. Heat Mass Transfer2007, 50, 405

[13] W. P. Pratt Jr., S. S. Rosenblum, W. A. Steyert, J. A. Barclay, Cryogenics 1977, 17, 689 [14] G. Lorusso, M. Jenkins, P. Gónzalez-Monje, A. Arauzo, J. Sesé, D. Ruiz-Molina, O. Roubeau, M. Evangelisti, Adv. Mater.2013, 25, 2984

[15] Both single-walled and multi-walled individual CNTs can show extremely high thermal conductivities, see, e.g.: a) P. Kim, L. Shi, A. Majumdar, P. L. McEuen, Phys. Rev. Lett.2001, 87, 215502; b) C. Yu, L. Shi, Z. Yao, D. Li, A. Majumbar, NanoLett.2005, 5, 1842

[16] J. Liu, A. G. Rinzler, H. J. Dai, J. H. Hafner, R. K. Bradley, P. J. Boul, A. Lu, T. Iverson, K. Shelimov, C. B. Huffman, F. Rodriguez-Macias, Y. S. Shon, T. R. Lee, D. T. Colbert, R. E. Smalley, Science1998, 280, 1253

[17] D. Tasis, N. Tagmatarchi, A. Bianco, M. Prato, Chem. Rev.2006, 106, 1105

[18] M. Maugey, W. Neri, C. Zakri, A. Derré, A. Pénicaud, L. Noé, M. Chorro, P. Launois, M. Monthioux, P. Poulin, J. Nanosci. Nanotechnol.2007, 7, 2633

[19] R. Verdejo, S. Lamoriniere, B. Cottam, A. Bismarck, M. Shaffer, Chem. Commun.2007, 513

[20]C. G. Salzmann, S. A. Llewellyn, G. Tobias, M. A. H. Ward, Y. Huh, M. L. H. Green, Adv. Mater.2007, 19, 883

[21] The simple mechanical mixture of MWCNT-ox and $\left[\mathrm{Gd}_{2} \mathrm{ac}\right]$ maintains the XRD pattern and the characteristic IR bands of [ $\left.\mathrm{Gd}_{2} \mathrm{ac}\right]$ (see Figure S5), both absent for the MWCNT$\left[\mathrm{Gd}_{2} \mathrm{ac}\right]$ composite.

[22] O. Roubeau, G. Lorusso, S. J. Teat, M. Evangelisti, Dalton Trans.2014, 43, 11502

[23] E. Natividad, M. Castro, R. Burriel, L. A. Angurel, J. Therm. Anal. Calor.2006, 2, 307 
[24] J. A. Barclay, L. Paterson, D. Bingham, O. Moze, Cryogenics1978, 18, 535

[25] W. F. Giauque, Phys. Rev.1933, 43, 0768

[26] G. A. Slack, D. W. Oliver, Phys. Rev. B1971, 4, 592

[27] as observed for $\mathrm{Gd}_{2}\left(\mathrm{SO}_{4}\right)_{3} \cdot 8 \mathrm{H}_{2} \mathrm{O}$, see reference 26

[28] J-H.Pöhls, M. B. Johnson, M. A. White, R. Malik, B. Ruff, C. Jayasinghe, M. J. Schulz, V. Shanov, Carbon2012, 50, 4175

[29] J. P. Small, L. Shi, P. Kim, Solid State Commun.2003, 127, 181

[30] M. B. Jakubinek, M. A. White, G. Li, C. Jayasinghe, W. Cho, M. J. Schulz, V. Shanov, Carbon2010, 48, 3947

[31] Z. Han, A. Fina, Prog. Polym. Sci.2011, 36, 914

[32] L. Zhang, G. Zhang, C. Liu, S. Fan, Nano Lett.2012, 12, 4848

[33] J. W. Sharples, D. Collison, E. J. L. McInnes, J. Schnack, E. Palacios, M. Evangelisti, Nat. Commun.2015, 5, 5321

[34] V. K. Pecharsky, K. A. Gschneidner, J. Appl. Phys.1999, 86, 565

[35] K. A. Wepasnick, B. A. Smith, K. E. Schrote, H. K. Wilson, S. R. Diegelmann, D. H. Fairbrother, Carbon2011, 49, 24 
Improved performance of molecular cryogenic coolers is achieved through a hybrid composite material with increased thermal conductivity. Direct measurements of the magnetocalorics combined with the determination of the thermal conductivity highlight the detrimental effect of the poor thermal conductivity of bulk molecular coolers and demonstrate the validity of a strategy using composite materials to resolve this drawback.

\section{Magnetocalorics}

O. Roubeau,* E. Natividad, M. Evangelisti, G. Lorusso, E Palacios

\section{A magnetocaloriccompositebased on} molecularcoolersandcarbonnanotubeswithenhanced thermal conductivity

ToC figure ((Please choose one size: $55 \mathrm{~mm}$ broad $\times 50 \mathrm{~mm}$ high or $110 \mathrm{~mm}$ broad $\times 20 \mathrm{~mm}$ high. Please do not use any other dimensions)) 
Supporting Information for the manuscript:

\section{A magnetocaloric composite based on molecular coolers and carbon nanotubes with enhanced thermal conductivity}

Olivier Roubeau, Eva Natividad, Marco Evangelisti, Giulia Lorusso, Elías Palacios

Table of contents

Figure S1. TEM images of raw MWCNTs

p. 2

Figure S2.Thermogravimetric analysis data

p.3

Figure S3.Powder X-ray diffractograms of MWCNT-ox and MWCNT-

p.4 $\left[\mathrm{Gd}_{2} \mathrm{ac}\right]$

Figure S4. Infra-red spectra of $\left[\mathrm{Gd}_{2} \mathrm{ac}\right]$ and MWCNT-[Gd $\mathrm{Gac}_{2}$

p. 5

Figure S5. Infra-red spectra of $\left[\mathrm{Gd}_{2} \mathrm{ac}\right]$ and physical mixture of MWCNT- p. 6 ox and $\left[\mathrm{Gd}_{2} \mathrm{ac}\right]$

Figure S6. Raman spectra of MWCNT-ox and MWCNT-[Gd $\mathrm{Gac}_{2} \quad$ p. 7

Figure S7.Powder X-ray diffractogram of the TGA residue of MWCNT- $\quad$ p.8 $\left[\mathrm{Gd}_{2} \mathrm{ac}\right]$

Figure S8.Comparison of thermogravimetric analysis of $\left[\mathrm{Gd}_{2} \mathrm{ac}\right]$ and p.9 MWCNT-[Gd $2 \mathrm{ac}]$

Table S1. Details and interpretation of thermogravimetric analysis p.10 results

Figure S9.Magnetization of the $\left[\mathrm{Gd}_{2} \mathrm{ac}\right]$ content in the composite p. 11 material

Figure S10. Magnetic entropy change of the $\left[\mathrm{Gd}_{2} \mathrm{ac}\right]$ content in the p. 12 composite material

$\begin{array}{ll}\left.\text { Figure S11. Heat capacity of bulk-[Gd}{ }_{2} \mathrm{ac}\right] & \text { p. } 13\end{array}$

$\begin{array}{ll}\text { Figure S12. Thermal conductivity of the LR White resin } & \text { p. } 14\end{array}$

$\begin{array}{ll}\text { Figure S13. Thermal conductance set-up and samples p. } 15 & \end{array}$

Table S2. Dimensions, weights and apparent densities of thermal p. 16 conductivity samples

Figure S14. Direct MCE measurement for a $m=0.81 \mathrm{mg}$ sample of $\quad$ p. 17 bulk-[Gd $\left.{ }_{2} \mathrm{ac}\right]$

Figure S15. Direct MCE measurement for a $m=8.1 \mathrm{mg}$ sample of bulk- $\quad$ p. 18 $\left[\mathrm{Gd}_{2} \mathrm{ac}\right]$

Figure S16. Direct MCE measurement for an array of parallel p. 19 buckypapers of MWCNT-[Gd $\left.{ }_{2} \mathrm{ac}\right]$

Modeling of thermal conductivity for the direct MCE measurements p. 20 

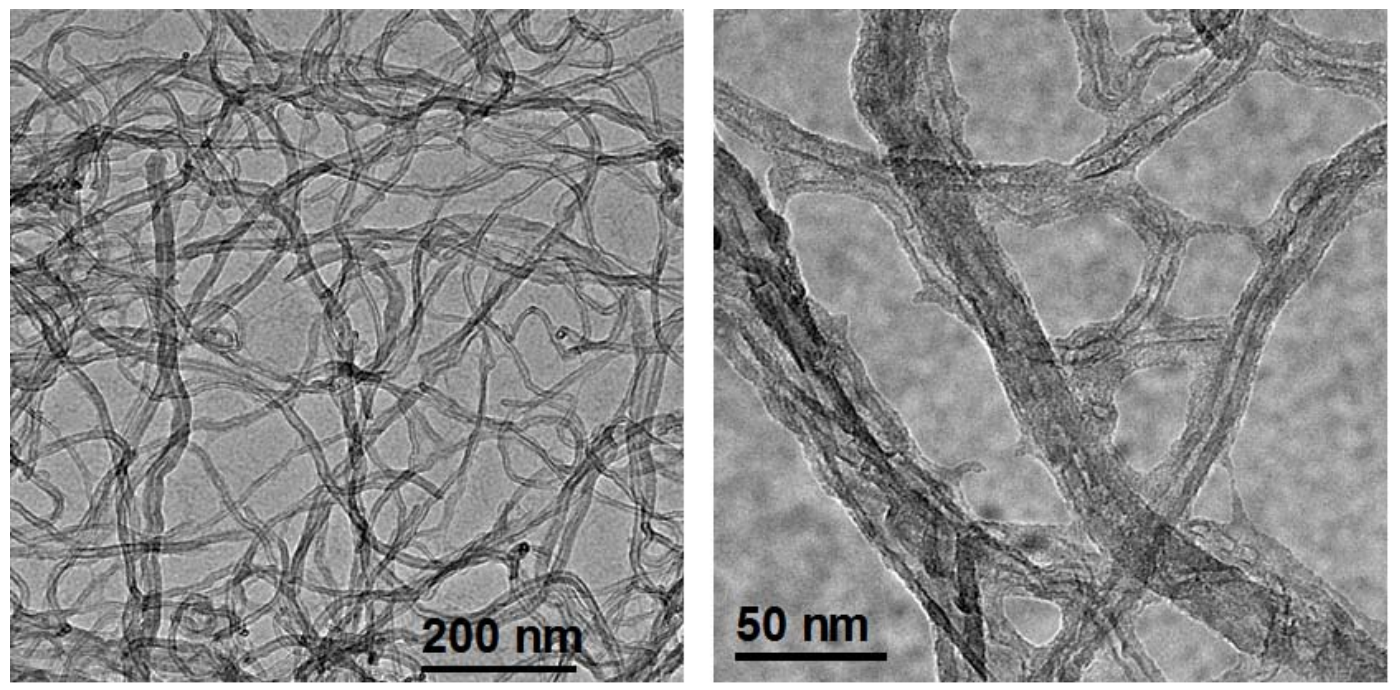

Figure S1. TEM images of the used raw MWCNTs after dispersion in water with sodium dodecyl sulphate and tip sonication. 


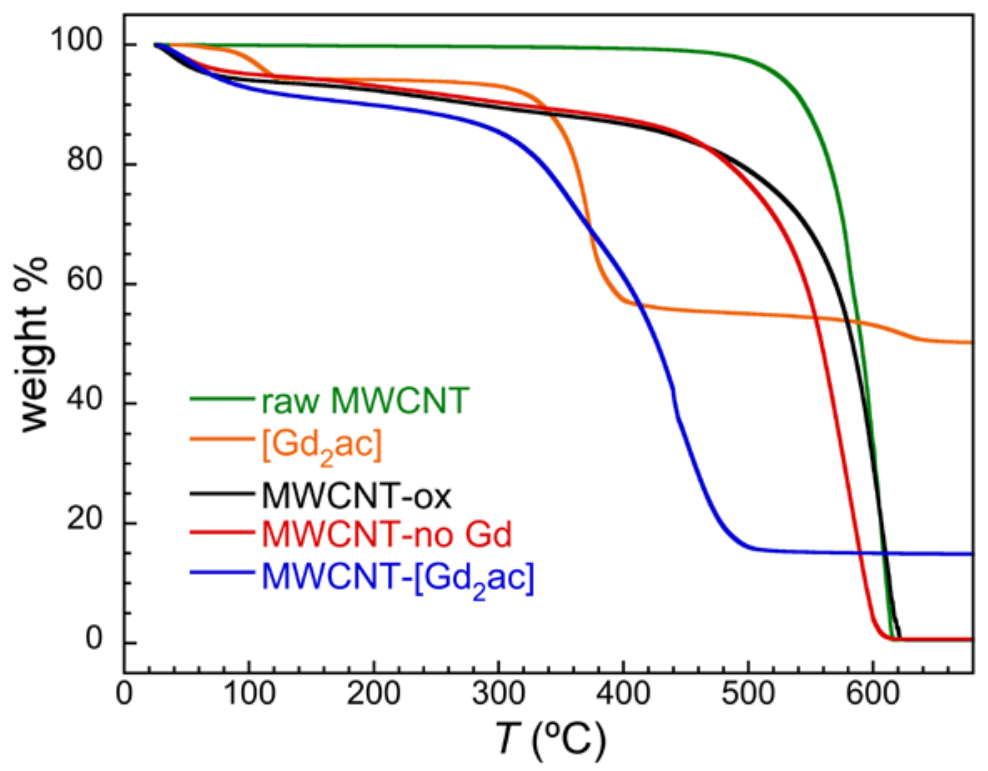

Figure S2. Thermogravimetric analysis under synthetic air performed at $10 \% \mathrm{~min}$ on the indicated materials. 


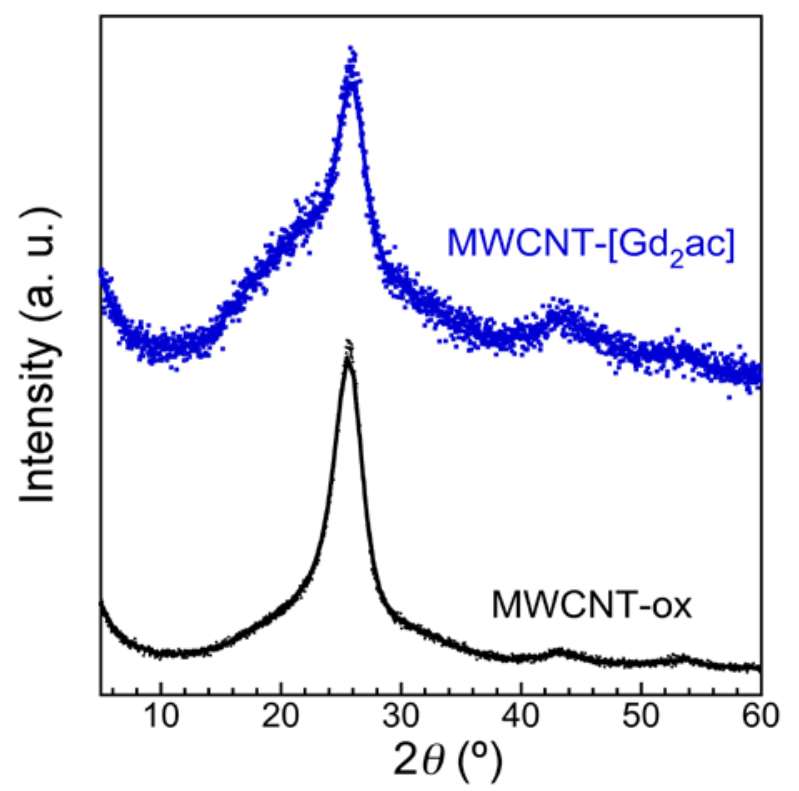

Figure S3. Powder X-ray diffractograms of bucky papers of MWCNT-ox and MWCNT-[Gd $\left[\mathrm{d}_{2} \mathrm{ac}\right]$, dominated by the peak at $c a$. $25.8^{\circ}$ characteristic of the 002 reflection from the graphitic layer structure of MWCNT, and corresponding to the inter-wall separation of ca. 3.4 $\AA$. Full lines are weighted smoothing of the experimental data (symbols). 

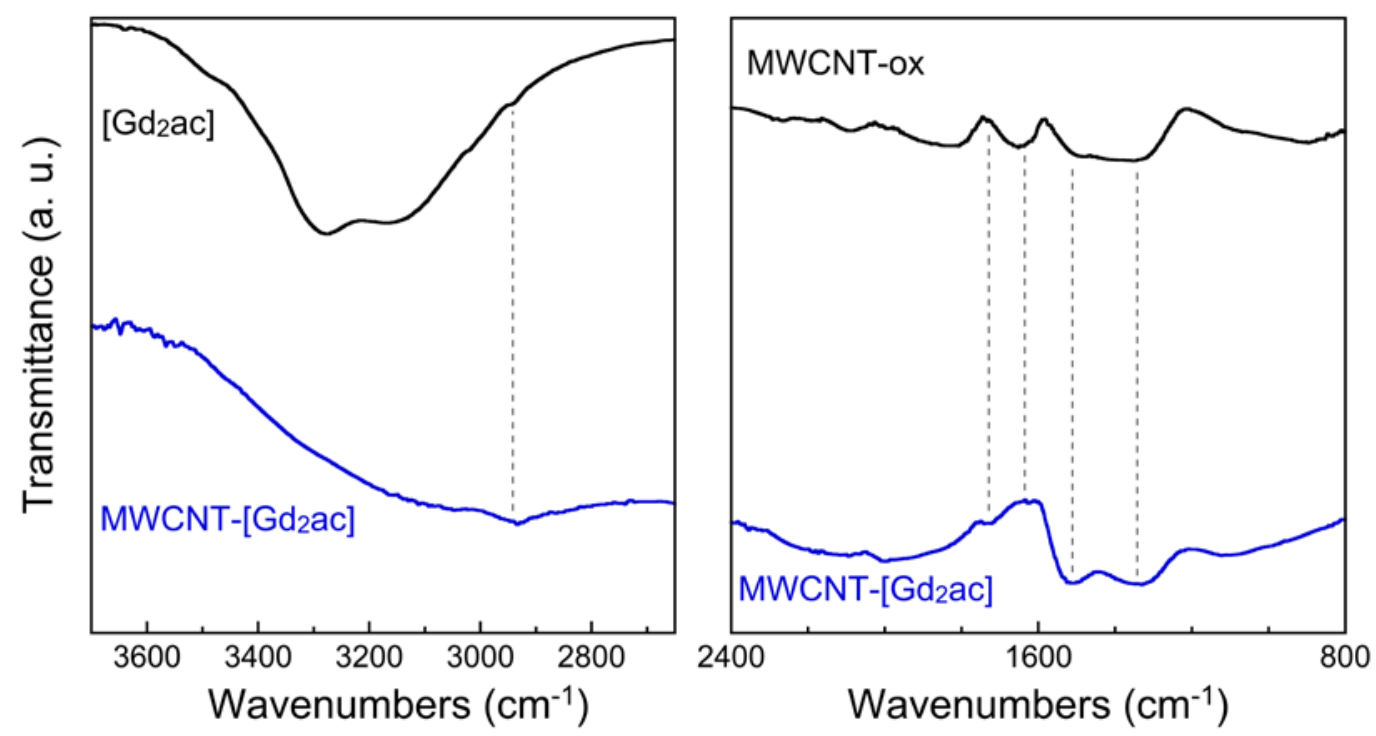

Figure S4. Infra-red spectra of $\left[\mathrm{Gd}_{2} \mathrm{ac}\right]$ and MWCNT-[ $\left[\mathrm{Gd}_{2} \mathrm{ac}\right]$. Vertical dashed lines highlight common features. 


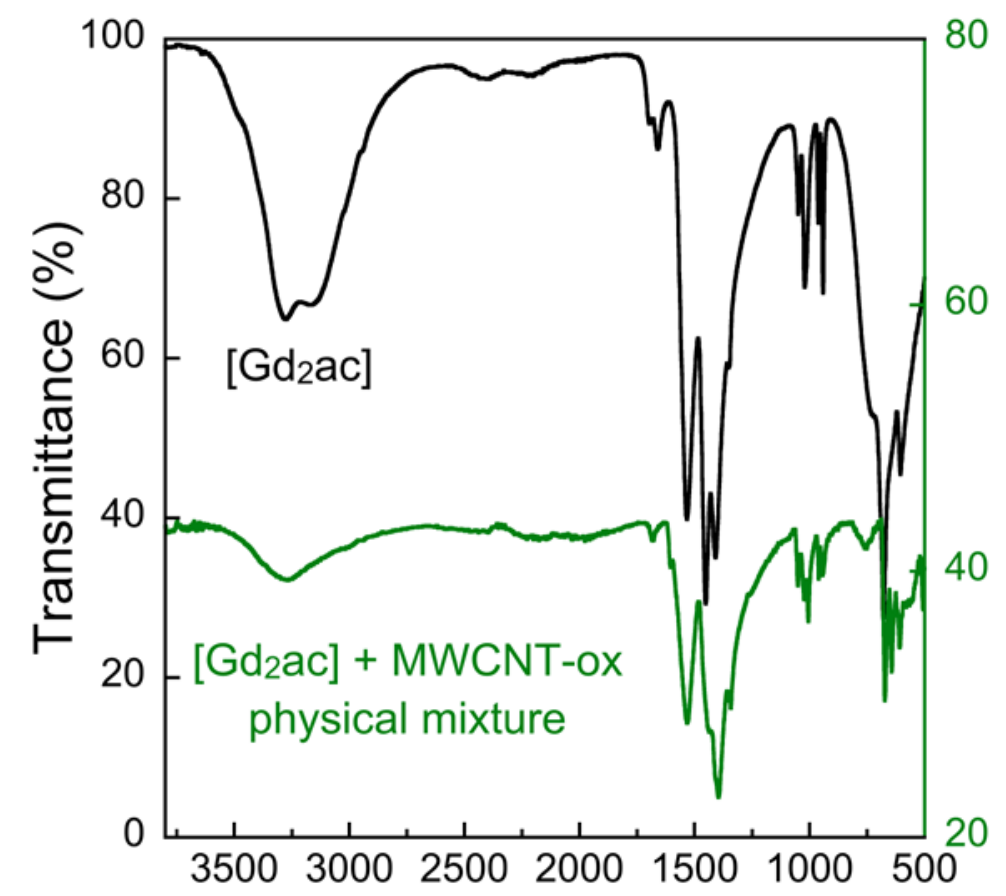

Figure S5. Infra-red spectra of $\left[\mathrm{Gd}_{2} \mathrm{ac}\right]$ and a physical mixture of MWCNT-ox and $\left[\mathrm{Gd}_{2} \mathrm{ac}\right]$. 


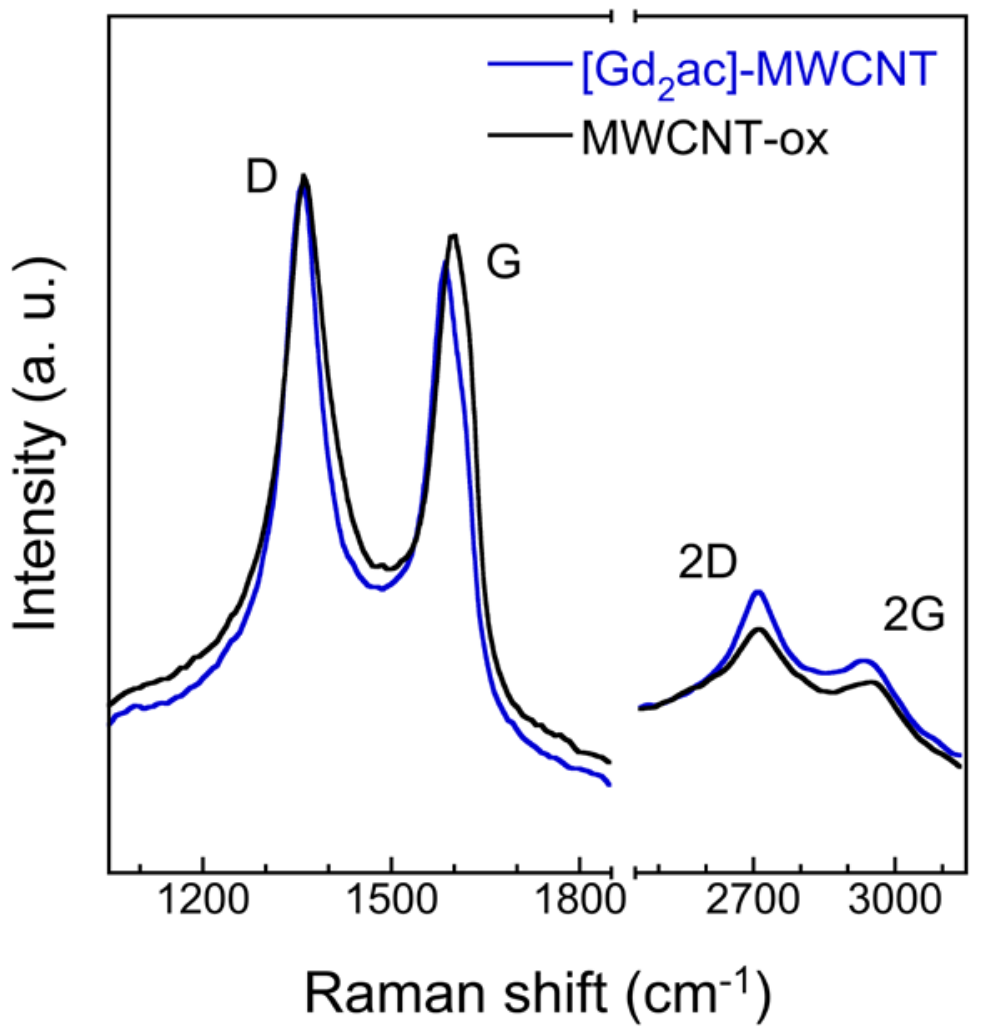

Figure S6. Raman spectra of MWCNT-ox and MWCNT-[Gd $\left.{ }_{2} a c\right]$. 


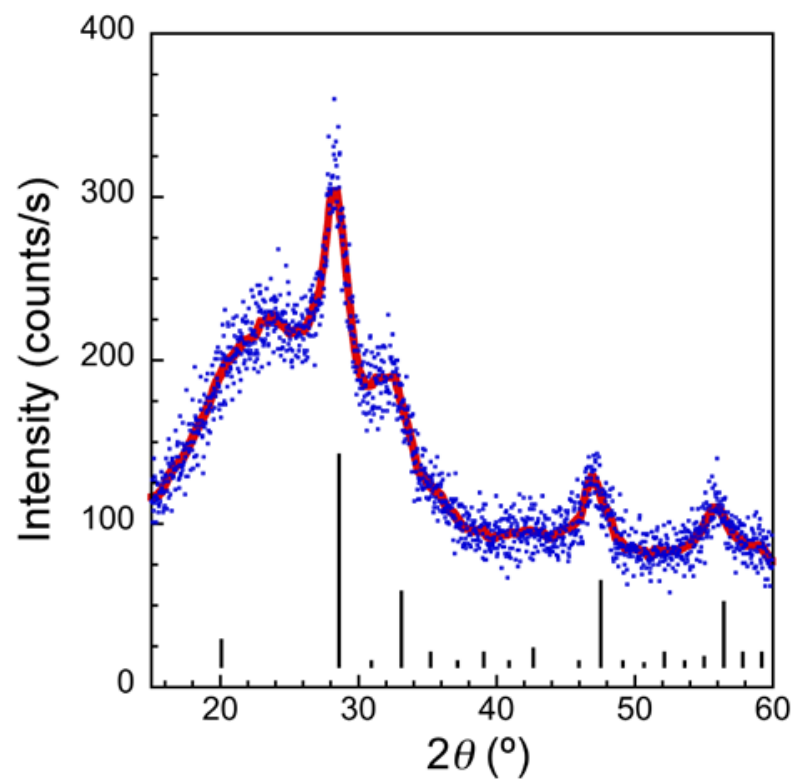

Figure S7. Powder X-ray diffractogram of the whitish residue after thermogravimetric analysis under synthetic air up to $700{ }^{\circ} \mathrm{C}$ of MWCNT-[Gd $\mathrm{ac}_{2}$. The full line is a weighted smoothing of the experimental data (symbols). Vertical bars represent the reflections of the cubic form of $\mathrm{Gd}_{2} \mathrm{O}_{3}$ (JCPDS 120797), the height being proportional to their relative intensity. 


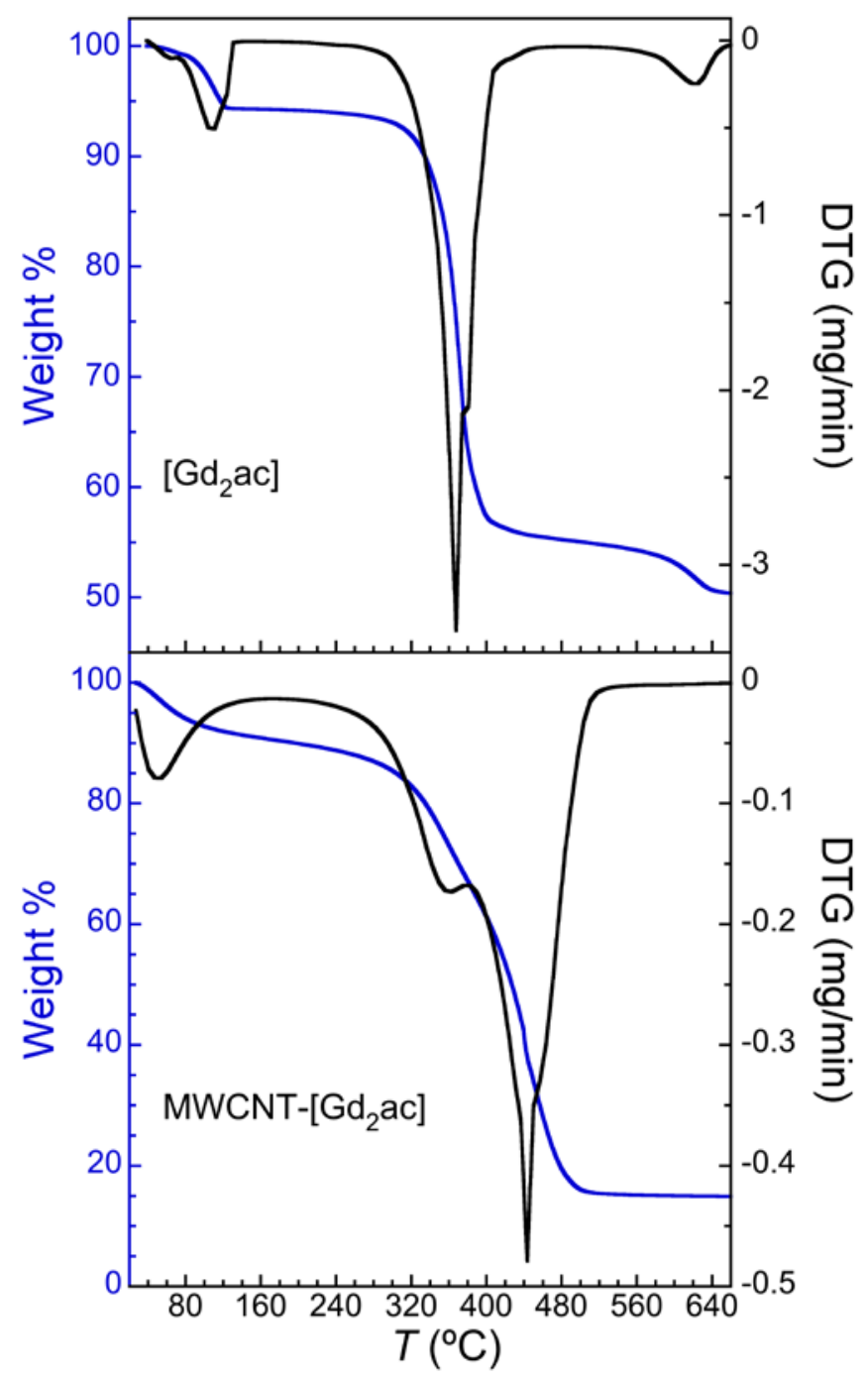

Figure S8. Comparison of the weight loss and its time derivative observed during thermogravimetric analysis under synthetic air for $\left[\mathrm{Gd}_{2} \mathrm{ac}\right]$ (top) and MWCNT-[Gd $2 \mathrm{ac}]$ (bottom). 
Table S1. Details and interpretation of thermogravimetric analysis results for raw MWCNT, MWCNT-ox, unreacted MWCNT-ox, MWCNT-[Gd $\left.\mathrm{Gac}_{2}\right]$ and $\left[\mathrm{Gd}_{2} \mathrm{ac}\right]$.

\begin{tabular}{|c|c|c|c|c|c|}
\hline Sample & $\begin{array}{c}\text { weight } \% \\
\left(T \text { in }{ }^{\circ} \mathrm{C}\right)\end{array}$ & $\begin{array}{l}\text { weight } \\
\text { loss (\%) }\end{array}$ & $\begin{array}{c}T \text { range } \\
\left({ }^{\circ} \mathrm{C}\right)\end{array}$ & $\begin{array}{l}T^{\text {peak }} \\
\left({ }^{\circ} \mathrm{C}\right)\end{array}$ & $\begin{array}{c}\text { Interpretation weight } \\
\text { loss }\end{array}$ \\
\hline \multirow[t]{2}{*}{ raw MWCNT } & $99.4(330)$ & 0.6 & $30-400$ & - & moist + defects \\
\hline & $0.5(620)$ & 98.1 & $400-620$ & 605 & oxidation CNT \\
\hline \multirow[t]{3}{*}{ MWCNT-OX } & $93.0(150)$ & 7.0 & $30-150$ & - & water \\
\hline & $87.1(390)$ & 5.9 & $150-390$ & 265 & oxygen-rich functions \\
\hline & $0.5(630)$ & 86.6 & $390-630$ & 607 & oxidation CNT \\
\hline unreacted & $94.3(150)$ & 5.7 & $30-150$ & - & water \\
\hline \multirow[t]{2}{*}{ MWCNT-ox } & $87.9(390)$ & 6.4 & $150-390$ & 265 & oxygen-rich functions \\
\hline & $0.6(630)$ & 87.3 & $390-630$ & 590 & oxidation CNT \\
\hline \multirow[t]{3}{*}{ MWCNT-[Gd $\left.{ }_{2} \mathrm{ac}\right]$} & $89.6(210)$ & 10.4 & $30-210$ & - & water \\
\hline & $61.9(400)$ & 27.7 & $210-400$ & 362 & $\begin{array}{c}\text { acetate to carbonate } \\
{\left[\mathrm{Gd}_{2} \mathrm{ac}\right]+\text { O-rich }} \\
\text { functions on CNT }\end{array}$ \\
\hline & $15.2(550)$ & 26.7 & $400-550$ & 443 & $\begin{array}{l}\text { oxidation CNT + } \\
\text { oxidation to } \mathrm{Gd}_{2} \mathrm{O}_{3}\end{array}$ \\
\hline \multirow[t]{3}{*}[\mathrm{Gd}_{2}\mathrm{ac}]{} & $94.3(130)$ & 5.7 & $30-130$ & 107 & water \\
\hline & $55.7(440)$ & 38.6 & $130-440$ & 368 & acetate to carbonate \\
\hline & $50.4(660)$ & 5.3 & $440-660$ & 625 & oxidation to $\mathrm{Gd}_{2} \mathrm{O}_{3}$ \\
\hline
\end{tabular}




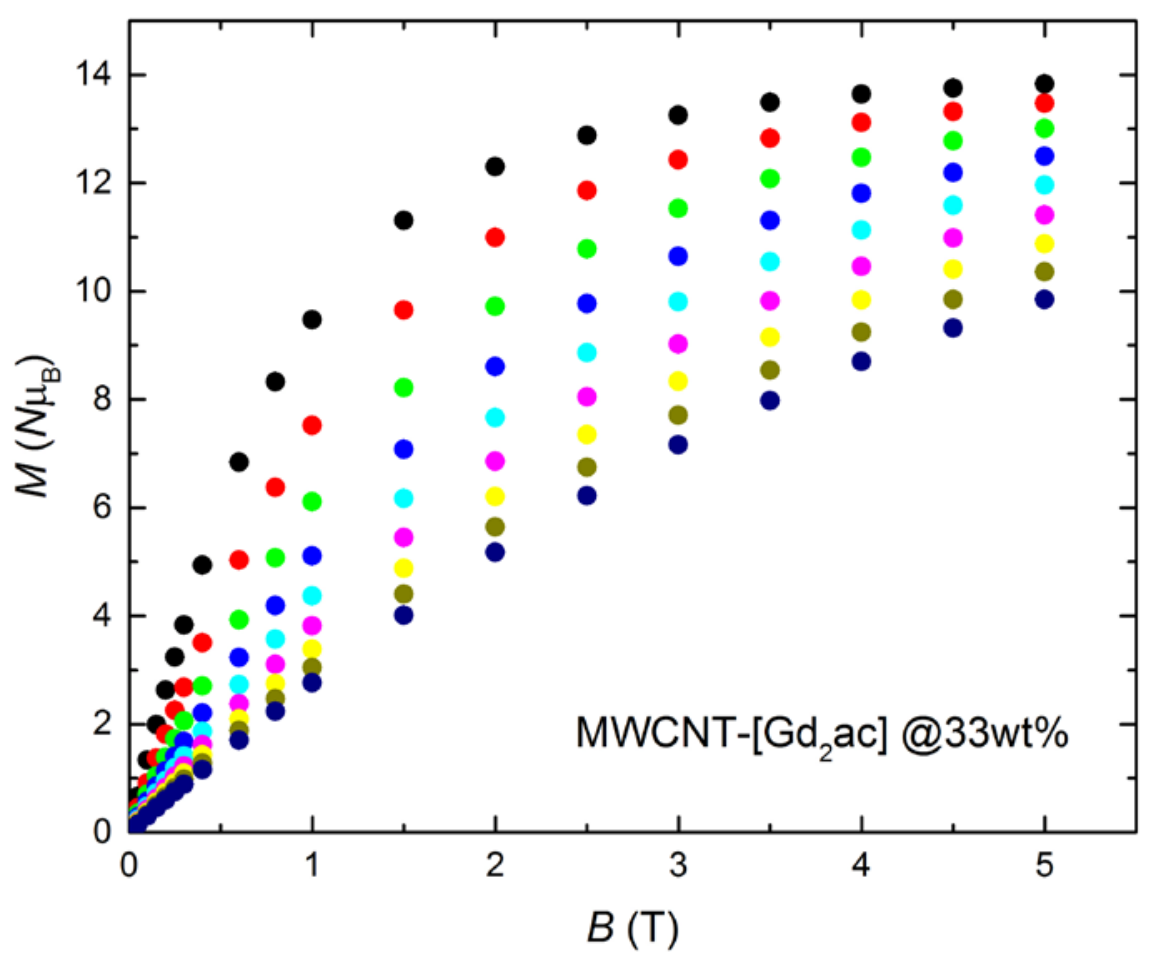

Figure S9. Magnetization of the $\left[\mathrm{Gd}_{2} \mathrm{ac}\right]$ content in the composite material MWCNT-[Gd $\mathrm{Gac}_{2}$ vs. applied field for temperatures in the range $2-10 \mathrm{~K}$. 


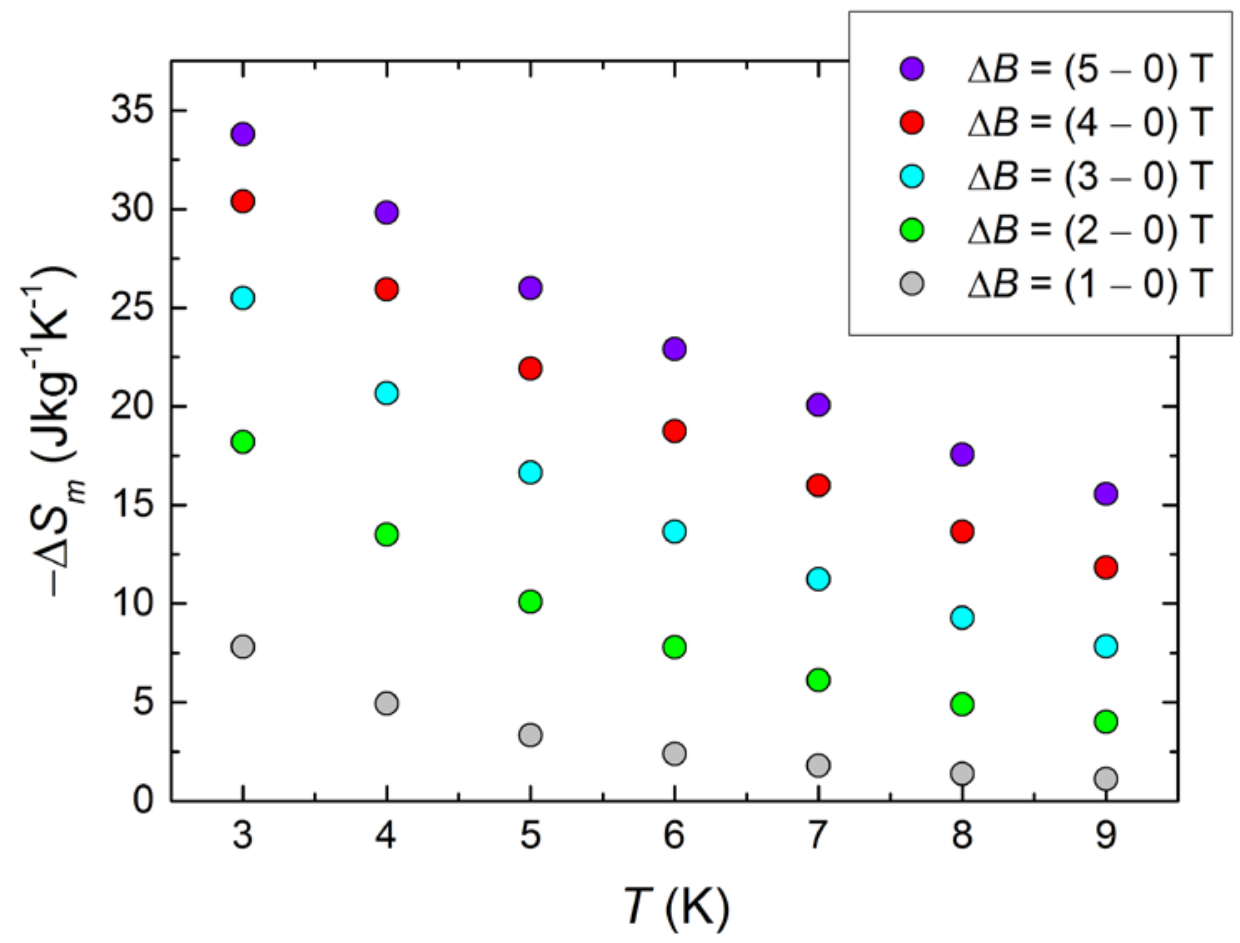

Figure S10. Temperature dependence of the magnetic entropy change $\Delta S_{\mathrm{m}}(T, \Delta B)$ at the indicated applied field-changes $\Delta B$, as derived indirectly from magnetization data (Figure S9). 


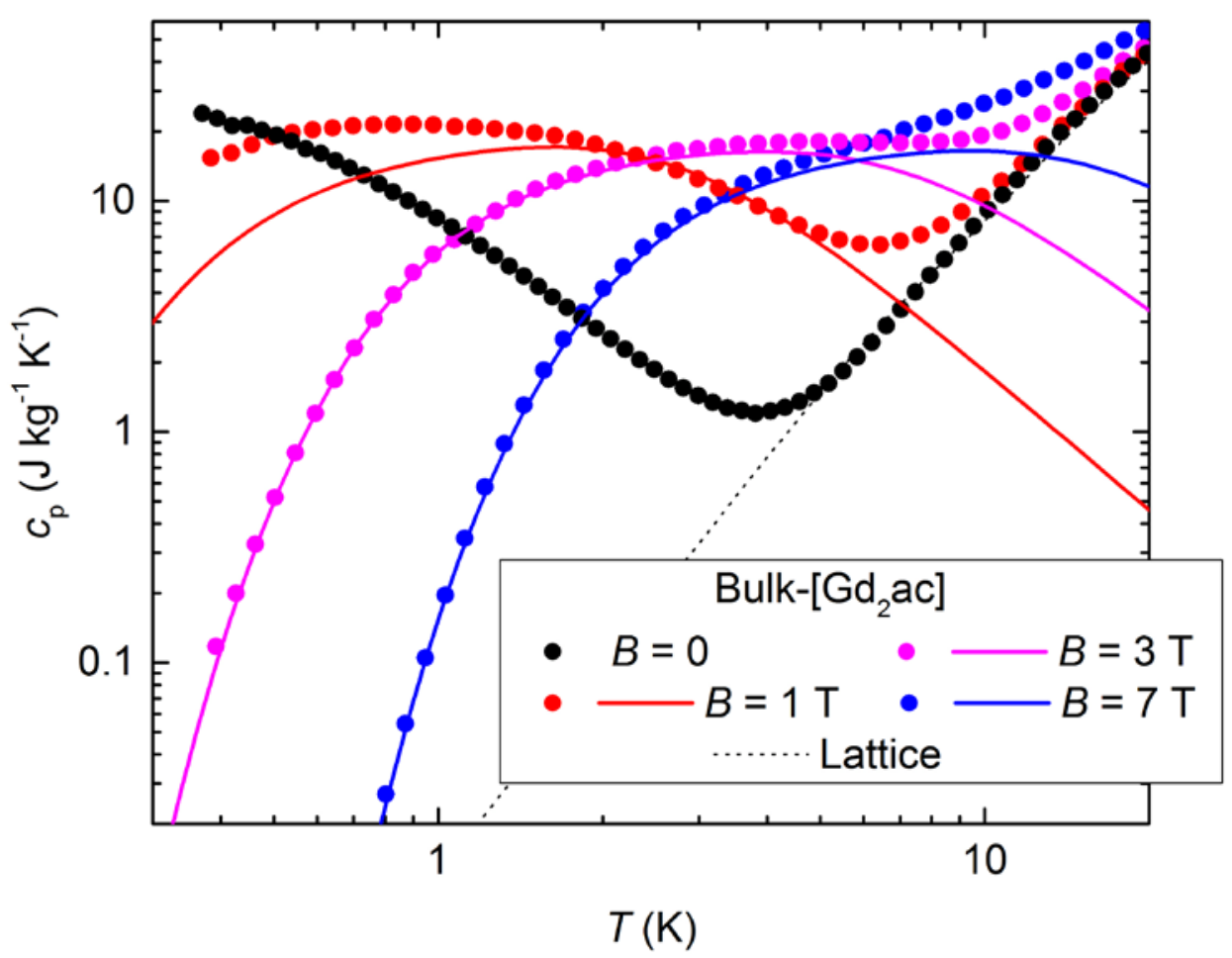

Figure S11. Temperature-dependence of the mass heat capacity of bulk$\left[\mathrm{Gd}_{2} \mathrm{ac}\right]$, for selected applied field values, as labeled. Solid lines are the Schottky heat capacities for $J=0.07 \mathrm{~K}$ (see text), while dashed line is the lattice contribution to the heat capacity. 


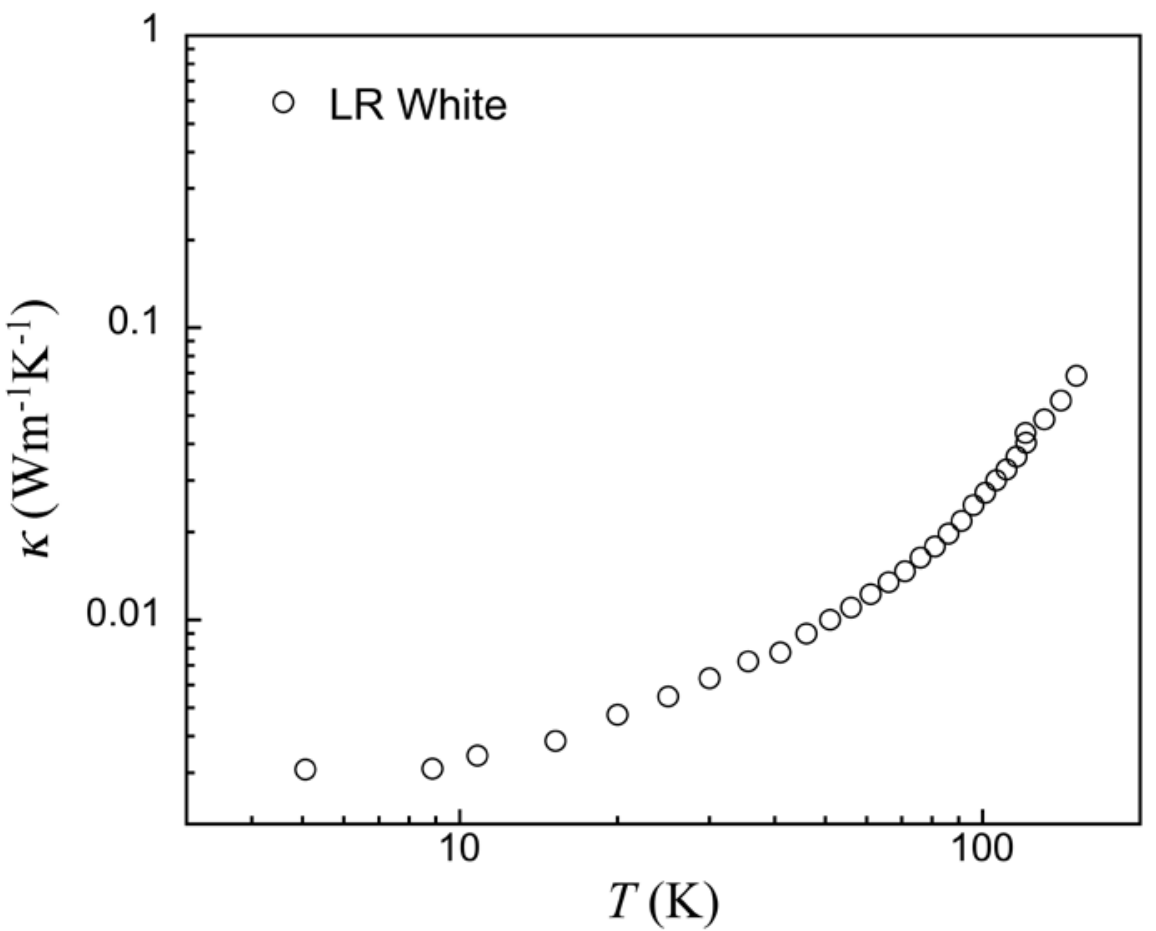

Figure S12.Thermal conductivity of the LRWhite acrylic resin for electron microscopy. 

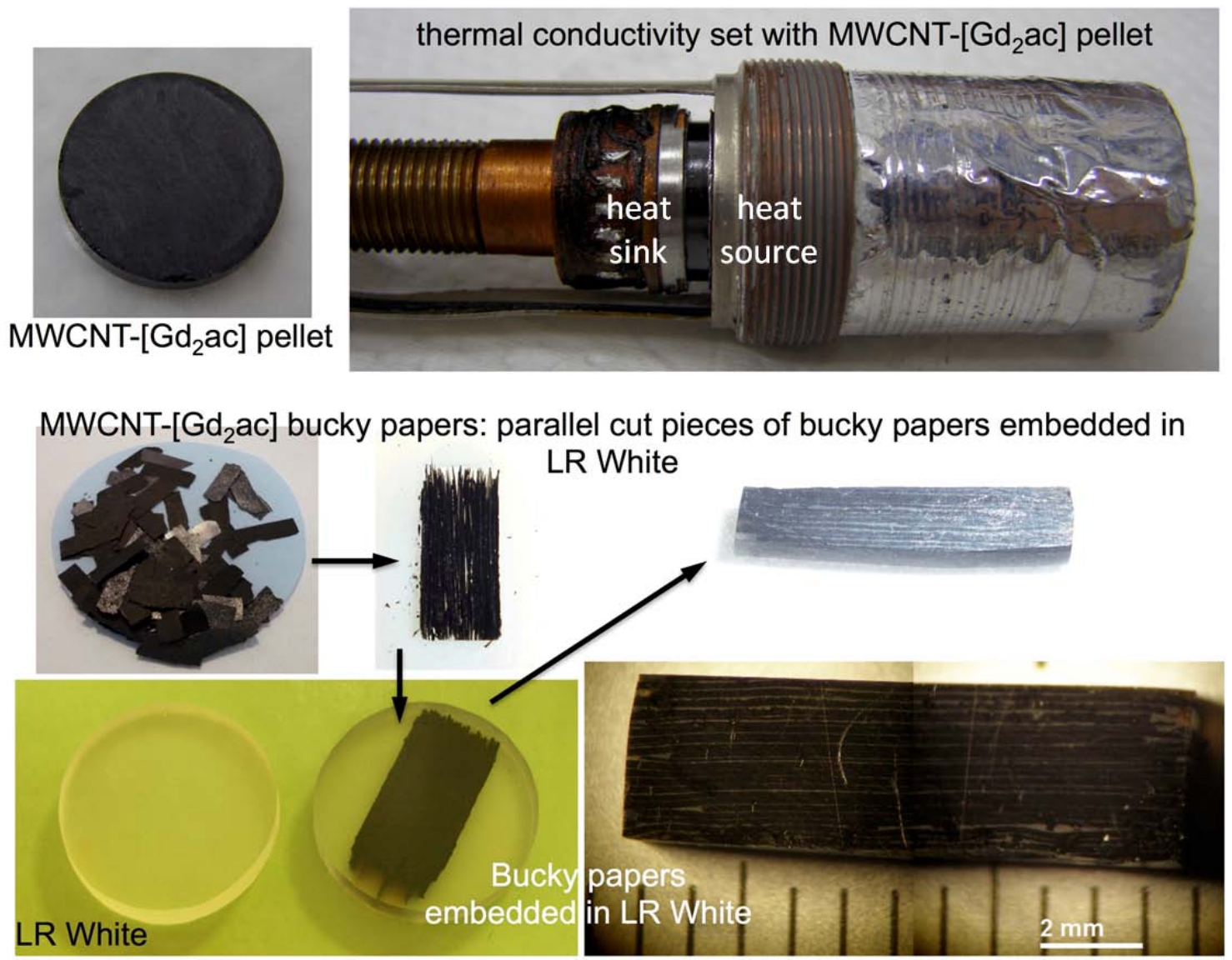

Figure S13. Details of the setup used for thermal conductance measurements and samples used for these measurements. 
Table S2. Dimensions, weights and derived apparent densities of the four samples used for the thermal conductivity study.

\begin{tabular}{|c|c|c|c|c|c|}
\hline Sample & mass (g) & area $\left(\mathrm{cm}^{2}\right)$ & height $(\mathrm{cm})$ & $V\left(\mathrm{~cm}^{3}\right)$ & $d_{\text {app }}\left(\mathrm{g} / \mathrm{cm}^{3}\right)$ \\
\hline [Gd $\left.{ }_{2} \mathrm{ac}\right]$ pellet & 1.10720 & 1.379 & 0.445 & 0.6136 & 1.804 \\
\hline MWCNT- $\left[\mathrm{Gd}_{2} \mathrm{ac}\right]$ pellet & 0.36666 & 1.379 & 0.165 & 0.2275 & 1.612 \\
\hline MWCNT-[Gd $\left.{ }_{2} a c\right]$ in LR White & 0.11495 & 0.3873 & 0.225 & 0.08715 & 1.319 \\
\hline LRWhite & 0.49864 & 1.735 & 0.2575 & 0.4467 & 1.116 \\
\hline
\end{tabular}




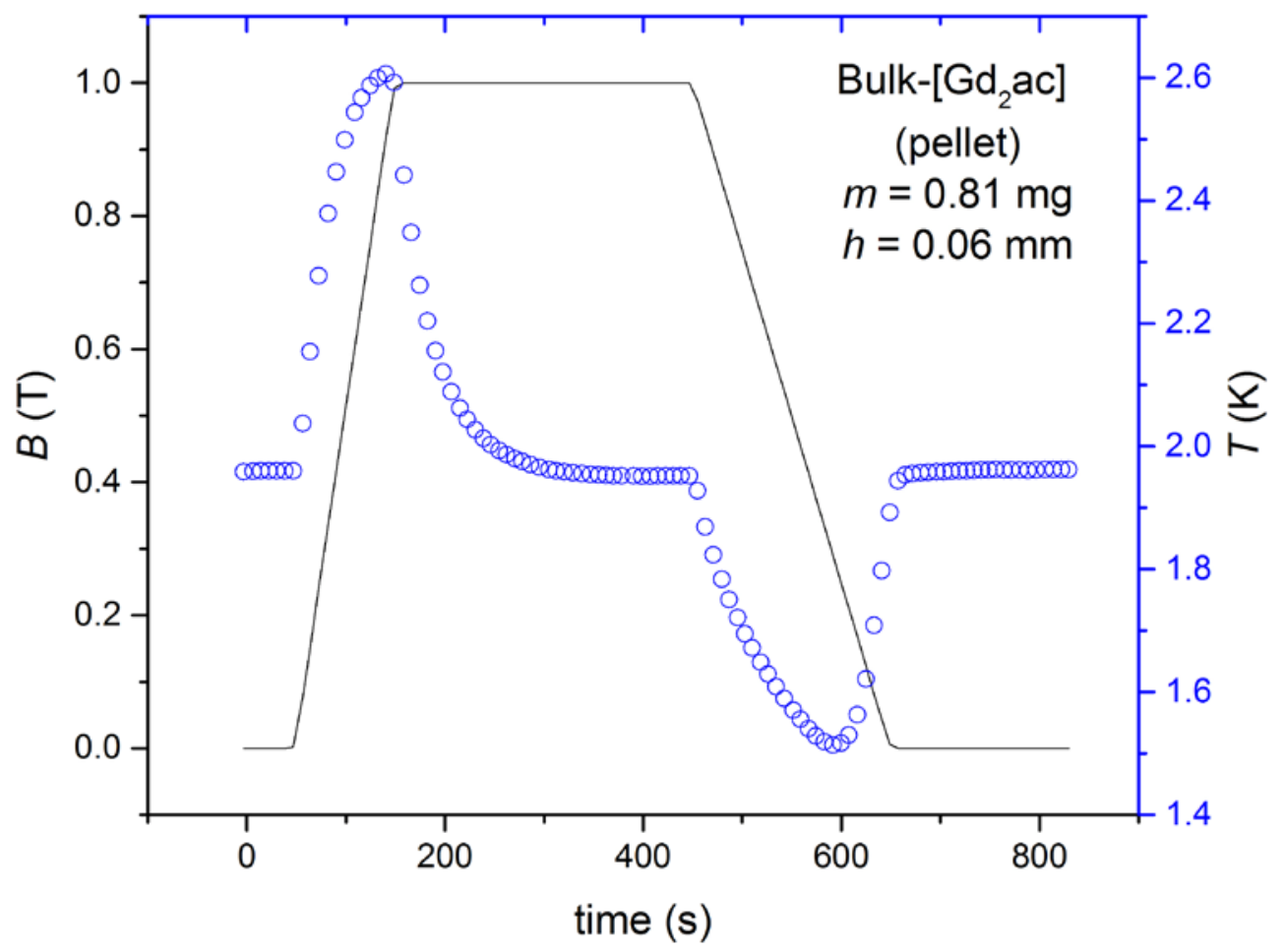

Figure S14. Direct measurement of the magnetocaloric effect for a pellet sample of bulk-[Gd $\mathrm{Gac}_{2}$ ( $m=0.81 \mathrm{mg}$ ). Line: time evolution of the applied field $B$ $(0 \rightarrow 1 \mathrm{~T} \rightarrow 0)$; points:experimental temperature $T$, as labeled, starting from $T=$ $1.96 \mathrm{~K}$. 


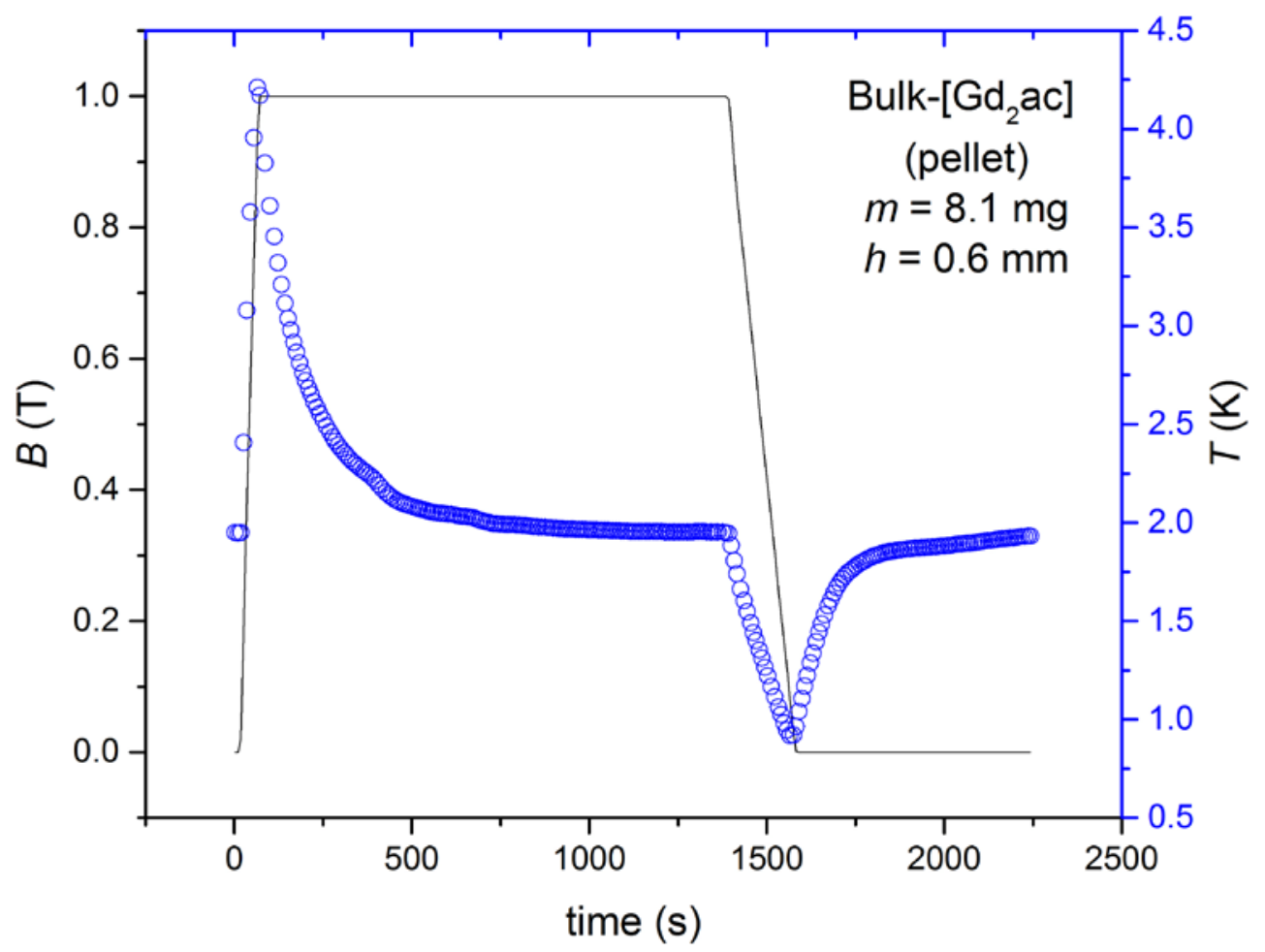

Figure S15. Direct measurement of the magnetocaloric effect for a pellet

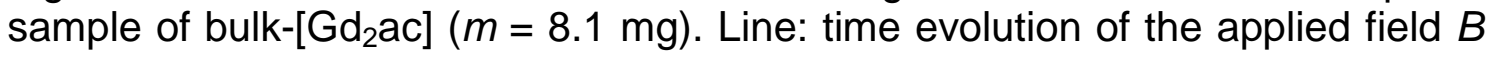
$(0 \rightarrow 1 \mathrm{~T} \rightarrow 0)$; points: experimental temperature $T$, as labeled, starting from $T=$ $1.96 \mathrm{~K}$. 


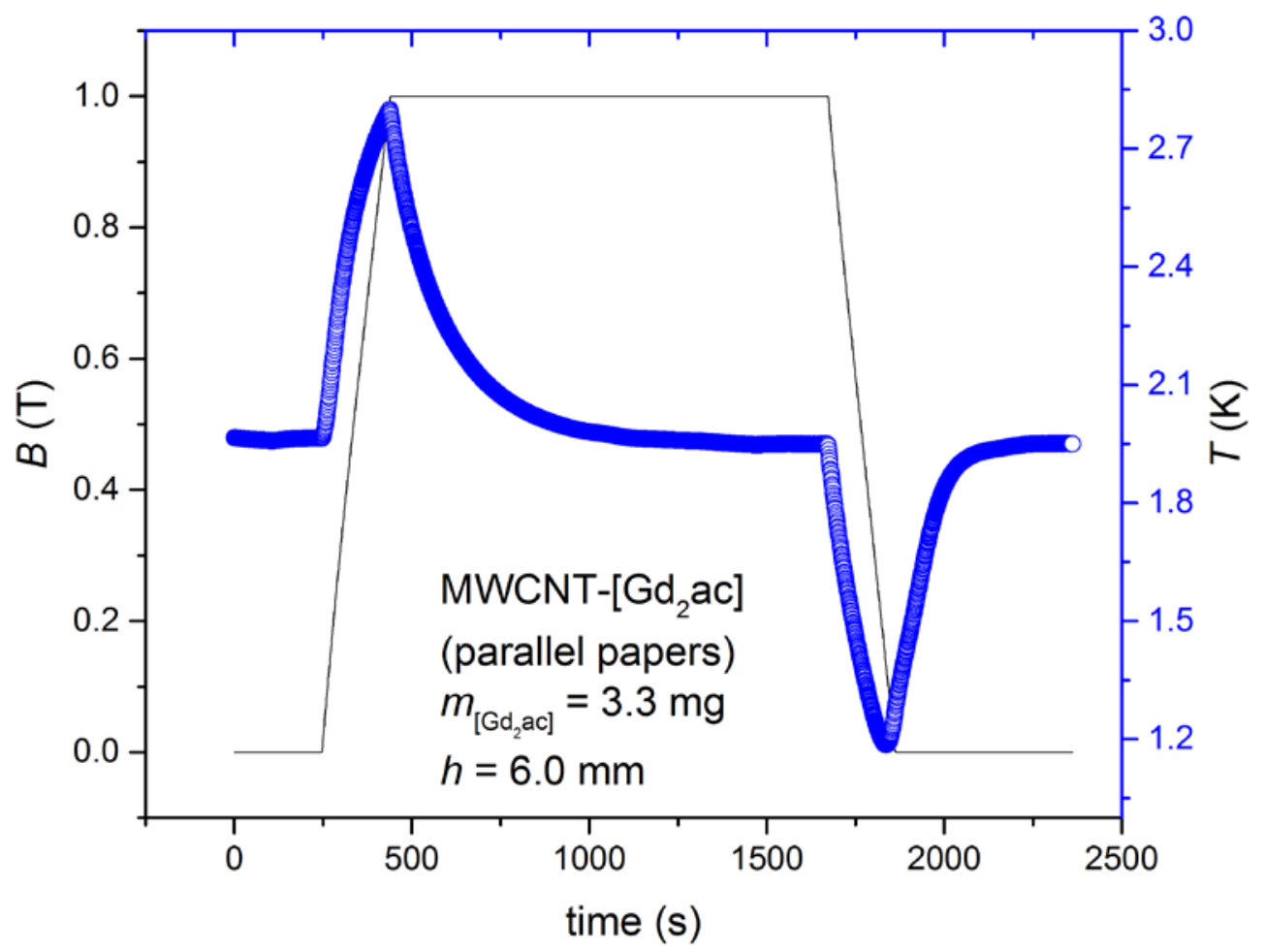

Figure S15. Direct measurement of the magnetocaloric effect for an array of parallel buckypapers of MWCNT-[Gd$a \mathrm{ac}]\left(m_{\text {[Gd2ac] }}=3.3 \mathrm{mg}\right.$, normalized per $\left.\left[\mathrm{Gd}_{2} \mathrm{ac}\right]\right)$. Line: time evolution of the applied field $B(0 \rightarrow 1 \mathrm{~T} \rightarrow 0)$; points: experimental temperature $T$, as labeled, starting from $T=1.96 \mathrm{~K}$. 
Modelling of the intrinsic thermal conductivity and direct MCE measurements

In our experiments, the cylindrically-shaped sample of bulk-[Gd$\left.{ }_{2} a c\right]$ lies on top of a sapphire platform (see scheme below), which is mechanically suspended and thermally connected to a thermal bath, hold at constant temperature $T_{0}$, by wires of known thermal $\operatorname{conductance}_{\mathrm{w}}(T)$. At liquid-helium temperatures, platform, thermometer and wires contribute negligibly to the heat capacity, which is then entirely determined by the sample. We assume that the temperature of the sample (a) is homogenous along any direction parallel to the sapphire platform, (b) has a gradient along $z$, i.e., the direction perpendicular to the sapphire platform.

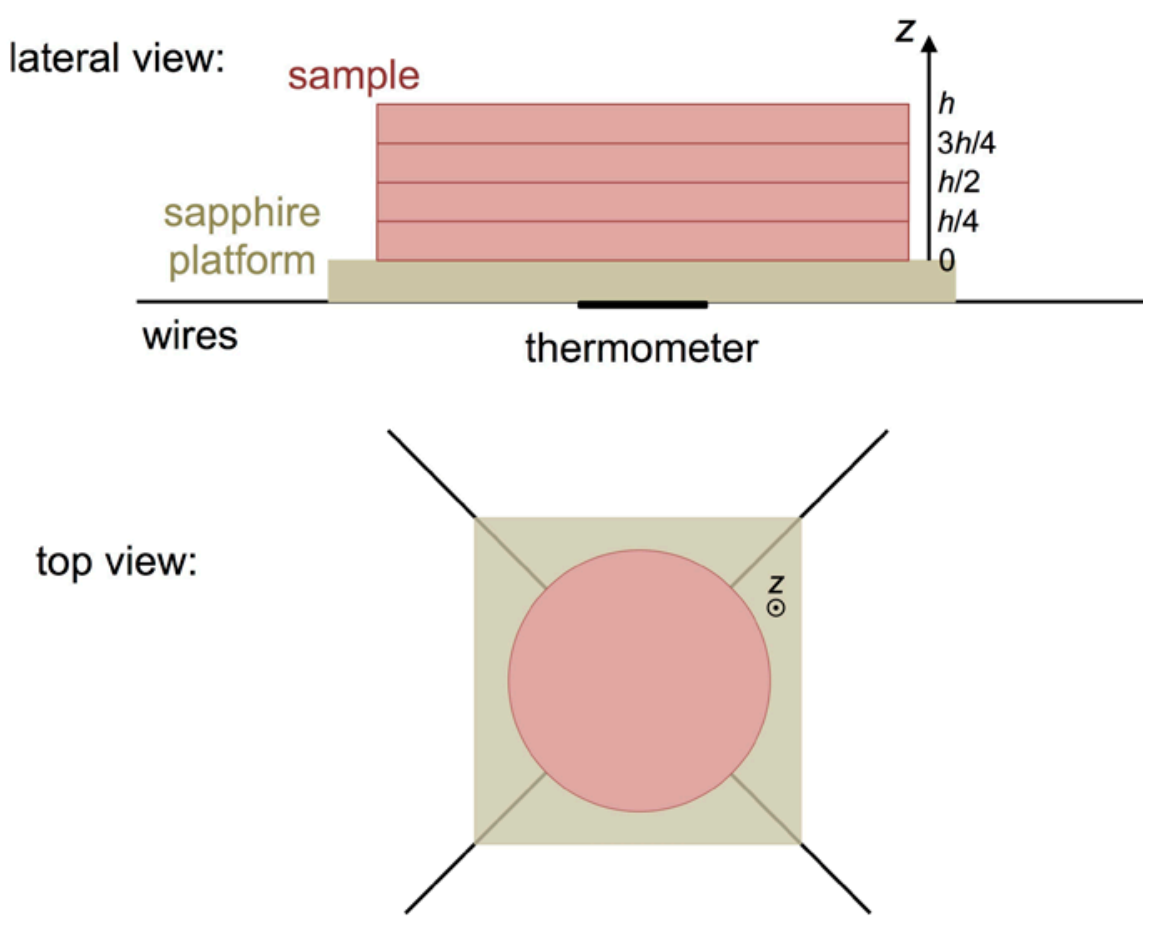

For any time-instant $t$ in which the applied magnetic field $B$ varies continuously, we simulate the evolution of the temperature, $T(t, z)$, across the sample. 
Specifically, we discretize the height, $h$, of the sample into 32 sections and calculate the temperature for each section, although only the following values of the heights are recorded as representative ones, namely $z=0$ (i.e., in contact with the platform), $h / 4, h / 2,3 h / 4$ and $h$. All relevant parameters are considered as data inputs in our simulation.

Next, we derive the equation which describes the heat conduction. Let us consider one mass unit of magnetocaloric material in the bore of a superconducting coil, which produces a magnetic field $B$. The workdone by an external source to hold a current in the superconducting coil is $d W=B d M$. Therefore, any infinitesimal change in energy can be expressed as $d U=d Q+d W=d Q+B d M$, according to the first law of thermodynamics. Let us further consider the magnetic enthalpy of a solid magnetic system when the pressure is negligible, i.e., $H=U-M B$. For any infinitesimal change, we thus obtain:

$$
d H=d U-M d B-B d M=d Q-M d B=T d S-M d B .
$$

Note that $d H$ can also be written in terms of $d T$ and $d B$, i.e.,

$$
\begin{aligned}
& d H=\left(\frac{\partial H}{\partial T}\right)_{B} d T+\left(\frac{\partial H}{\partial B}\right)_{T} d B=c_{p} d T+\left(\frac{T d S-M d B}{d B}\right)_{T} d B \\
& =c_{p} d T+T\left(\frac{\partial S}{\partial B}\right)_{T} d B-M d B
\end{aligned}
$$

where $c_{p}$ is the heat capacity of the sample under constant applied magnetic field. By equating (1) to (2), we obtain:

$$
d Q=c_{p} d T+T\left(\frac{\partial S}{\partial B}\right)_{T} d B .
$$


The heat gained through conductionby any volume $V$ of the magnetocaloric material in an infinitesimal time interval $d t$ is given by

$$
\begin{aligned}
& d Q=-d t \oint_{A} \mathbf{J}_{Q} \cdot d \mathbf{A}=d t \oint_{A} \kappa \nabla T \cdot d \mathbf{A}=d t \int_{V} \nabla \cdot(\kappa \nabla T) d V \\
& =d t \int_{V}\left(\nabla \kappa \cdot \nabla T+\kappa \nabla^{2} T\right) d V
\end{aligned}
$$

where $J_{Q}$ is the heat flux density, $A$ is the surface enclosing $V$, and $\kappa$ is the intrinsic thermal conductivity of the sample.In (4) we make use of the Fourier law and the divergence theorem. The intrinsic thermal conductivity is included in our simulations by fitting the experimental data in Figure 4 to a $\kappa=\alpha T^{n}$ dependence at low temperatures. The gradient $\nabla \kappa$ can then be expressed as

$$
\nabla \kappa=\frac{d \kappa}{d T} \nabla T=n \alpha T^{n-1} \nabla T=\frac{n \kappa}{T} \nabla T
$$

Moreover, for any small volume $V$ the integrand in (4) can be considered constant and this equation simplifies to

$$
d Q=V d t\left[\frac{n \kappa}{T}(\nabla T)^{2}+\kappa \nabla^{2} T\right]
$$

Replacing $d Q$ in (3), for mass $\rho V$, where $\rho$ is the density, we obtain:

$$
\kappa V d t\left[\frac{n}{T}(\nabla T)^{2}+\nabla^{2} T\right]=\rho V c_{p} d T+\rho V T\left(\frac{\partial S}{\partial B}\right)_{T} d B
$$

or, equivalently,

$$
d T=\frac{\kappa d t}{\rho c_{p}}\left[\frac{n}{T}(\nabla T)^{2}+\nabla^{2} T\right]-\frac{T}{c_{p}}\left(\frac{\partial S}{\partial B}\right)_{T} d B .
$$


This is the master equation commonly employed for the simulation of heat transfer in prototypes of magnetocaloric refrigerators. ${ }^{1}$ The last term in (5) can be transformed using the exact thermodynamic relation

$$
-\frac{T}{c_{p}}\left(\frac{\partial S}{\partial B}\right)_{T}=\left(\frac{\partial T}{\partial B}\right)_{S},
$$

thus, yielding:

$$
d T=\frac{d t}{\rho c_{p}}\left[\frac{n \kappa}{T}(\nabla T)^{2}+\kappa \nabla^{2} T\right]+\left(\frac{\partial T}{\partial B}\right)_{S} d B
$$

Finally, dividing each term by $d t$ and considering that, in one dimension, the temperature $T(t, z)$ is a function of time $t$ and of a single space coordinate $z$, we obtain:

$$
\frac{\partial T}{\partial t}=\frac{\kappa}{\rho c_{p}}\left[\frac{n}{T}\left(\frac{\partial T}{\partial Z}\right)^{2}+\left(\frac{\partial^{2} T}{\partial Z^{2}}\right)\right]+\left(\frac{\partial T}{\partial B}\right)_{S} \frac{d B}{d t} .
$$

Note that $\left(\frac{\partial T}{\partial B}\right)_{S}$ is relatively simple to implement in a numerical calculation, thus minimizing discretization errors. For instance in the present case, we deal with a nearly paramagnetic material, for which $\left(\frac{\partial T}{\partial B}\right)_{S} \cong \frac{T}{B}$. Equation (7) is finally solved numerically by using the Crank-Nicolson method. ${ }^{2}$

Furthermore, we determine the adiabatic temperature $T_{a d}$, viz., the temperature if the sample would have been kept thermally isolated, using the same method

\footnotetext{
${ }^{1}$ See, e.g., K. K. Nielsen, J. Tusek, K. Engelbrecht, S. Schopfer, A. Kitanovski, C.R.H. Bahl, A. Smith, N. Pryds, A. Poredos, Int. J. Refrig.2011, 34 603-616

${ }^{2}$ J. Crank, P. Nicolson, Proc. Camb. Phil. Soc.1947, 43, 50-67
} 
we employed for other works. ${ }^{3}$ Briefly, we note that the non-adiabaticity induces a variation of the entropy $\Delta S=S(t)-S\left(t_{0}\right)$ in a time interval $t-t_{0}$, which can be expressed as $\Delta S=\int_{t_{0}}^{t} K_{w}\left(T_{0}-T\right) / T d t$ at every time instant. We also have $\Delta S=\int_{T_{a d}}^{T} C_{p}(T, B) / T d T$, where the adiabatic temperature $T_{a d}$ is the only unknown and can therefore be deduced numerically.

The results of the simulation are compared with the experimental temperatures collected for two pellet samples of bulk-[Gd $\left.\mathrm{Gac}_{2}\right]$ with a spherical-shaped base, $h=0.06$ and $0.6 \mathrm{~mm}$, respectively (Figures 5 and 6), and for an array of parallel buckypapers of MWCNT-[Gd $\left.\mathrm{Gd}_{2} \mathrm{ac}\right]$ with rectangular-shaped base and $h=$ 6.0mm(Figure 6).

${ }^{3}$ See, e.g., J. W. Sharples, D. Collison, E. J. L. McInnes, J. Schnack, E. Palacios, M. Evangelisti, Nat. Commun.2014,5, 5321 